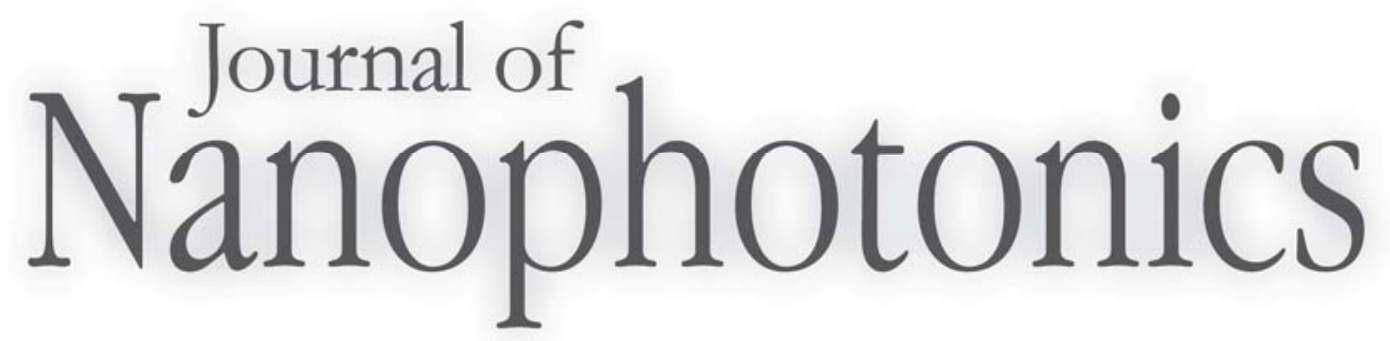

SPIEDigitalLibrary.org/jnp

\title{
Light-driven artificial molecular machines
}

Yue Bing Zheng

Qingzhen Hao

Ying-Wei Yang

Brian Kiraly

I-Kao Chiang

Tony Jun Huang 


\title{
Light-driven artificial molecular machines
}

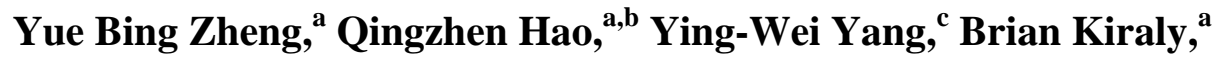 \\ I-Kao Chiang, ${ }^{a}$ and Tony Jun Huang ${ }^{a}$ \\ ${ }^{a}$ The Pennsylvania State University, Department of Engineering Science and Mechanics, \\ University Park, Pennsylvania 16802 \\ junhuang@psu.edu \\ ${ }^{\mathrm{b}}$ The Pennsylvania State University, Department of Physics, \\ University Park, Pennsylvania 16802 \\ ${ }^{\mathrm{c}}$ University of California, Irvine, Department of Chemistry, \\ 1102 Natural Sciences 2, Irvine, California 92697
}

\begin{abstract}
Artificial molecular machines represent a growing field of nanoscience and nanotechnology. Stimulated by chemical reagents, electricity, or light, artificial molecular machines exhibit precisely controlled motion at the molecular level; with this ability molecular machines have the potential to make significant impacts in numerous engineering applications. Compared with molecular machines powered by chemical or electrical energy, light-driven molecular machines have several advantages: light can be switched much faster, work without producing chemical waste, and be used for dual purposes-inducing (writing) as well as detecting (reading) molecular motions. The following issues are significant for light-driven artificial molecular machines in the following aspects: their chemical structures, motion mechanisms, assembly and characterization on solid-state surfaces. Applications in different fields of nanotechnology such as molecular electronics, nano-electro-mechanical systems (NEMS), nanophotonics, and nanomedicine are envisaged.
\end{abstract}

Keywords: artificial molecular machines, molecular electronics, NEMS, nanophotonics, plasmonics, nanomedicine

\section{INTRODUCTION}

The human body has numerous biologically inherited molecular motors and machines [1]. In these biomotors, nature elegantly demonstrates that simple molecular components can efficiently link motion from the nanometer scale to macroscopic scales and achieve complex functions once organized and assembled in a precise manner. These functions include, but are not limited to: powering skeletal muscles, synthesizing Adenosine-5'-triphosphate (ATP, the energy source for most living creatures), and producing DNA/RNA (the fingerprint of life). For example, the contraction and the extension of muscles takes place because of mutual sliding myosin fibers (polymer of biomotors) and actin filaments. When appropriately oriented and organized, assemblies of these myosin/actin nanocomposite materials can give rise to substantial macroscopic movements in human muscles.

Compared to biomotors, artificial molecular machines have a much shorter history $[2,3]$. Artificial molecular machines were first envisioned by Richard Feynman, one of the greatest physicists and teachers of the twentieth century. In a historic address [4], he contemplated, "What are the possibilities of constructing molecular-scale mechanical machines ... What would be the utility of such machines? Who knows? I cannot see exactly what would happen, but I can hardly doubt that when we have some control of the arrangement of things on a molecular scale we will get an enormously greater range of possible properties that substances can have, and of the different things we can do." In the 1980s, the earliest examples of artificial molecular machines were reported, with most of them based on the photoisomerization of azobenzenes [5]. Since then, the development of artificial molecular 
machines has become one of the most intriguing topics in the field of nanoscience and nanotechnology. Artificial molecular machines with various well-defined mechanical motions have been developed in the research labs around the world [6-27]. Figure 1 shows a schematic representation of some basic motions in molecular machines.

a

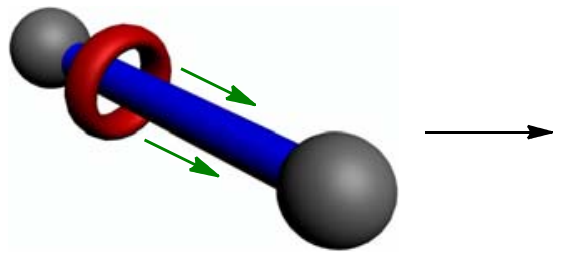

b

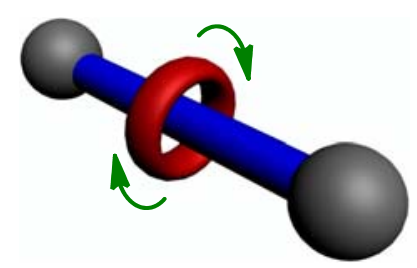

C

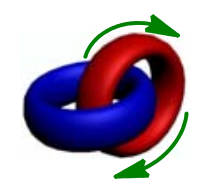

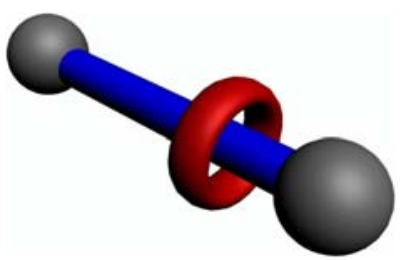

d

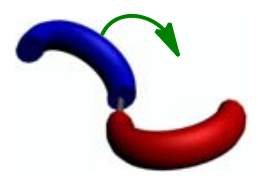

Fig. 1. Schematic representation of some basic movements of molecular machines: (a) linear motion; $(\mathrm{b}, \mathrm{c}, \mathrm{d})$ rotary motions.

All machines need power supplies to drive their motions; their function is to convert the supplied energy into mechanical motion to do work. As macroscopic machines require macroscopic energy sources, artificial molecular machines require drastically reduced amounts of energy to undergo directed molecular motions [3, 28-32]. Three types of energy sources - chemical [33-35], electrochemical [36], and photochemical [37]- have been employed to induce well-defined mechanical movements within molecular machines. Light, or photochemical processes, have several advantages over chemical and electrochemical stimuli in driving artificial molecular machines: light can function at much higher frequencies, act without producing chemical waste, and be used in a dual capacity - inducing (writing) as well as detecting (reading) molecular motions [29, 32, 38-40]. Progress in "bottom-up" synthetic chemistry has led to a variety of light-driven molecular machines [38]. In this review, we discuss the light-driven artificial molecular machines in the following aspects: chemical structures and functions (Sec. 2), assembly on solid-state surfaces (Sec. 3), characterization on solid-state surfaces (Sec. 4), and applications (Sec. 5).

\section{CHEMICAL STRUCTURES AND FUNCTIONS OF LIGHT-DRIVEN MOLECULAR MACHINES}

Figure 2 shows a multicomponent, bistable rotaxane $\mathbf{1}^{6+}$ that has been designed to work as a light-driven molecular machine based on photo-induced electron transfer reactions (its mechanical schematic is shown in Fig. 1a). The bistable rotaxane is composed of an electronrich macrocycle $(\mathbf{M})$ and a dumbbell-shaped component. The dumbbell-shaped component contains a $\mathrm{Ru}(\mathrm{II})$-bipyridine-based light-harvesting unit $\left(\mathbf{P}^{2+}\right)$ with a p-terphenyl-type ring system acting as a rigid spacer which separates the $\mathbf{P}^{2+}$ unit from the mechanical switching moiety. The mechanical switching moiety is composed of a $4,4^{\prime}$-bipyridinium component 
$\left(\mathbf{A 1}^{2+}\right)$ as a strong primary $\pi$-electron accepting unit, a 3,3'-dimethyl-4,4'-bipyridinium component $\left(\mathbf{A} 2^{2+}\right)$ as a weak secondary $\pi$-electron accepting unit, and a tetraarylmethane group (T). The $\mathbf{A} \mathbf{1}^{2+}$ and $\mathbf{A} \mathbf{2}^{2+}$ components act as stations for the macrocycle $\mathbf{M}$. $\mathbf{M}$ encircles the $\mathbf{A 1}^{2+}$ station in the starting state and can be switched mechanically between the $\mathbf{A 1}^{2+}$ and the $\mathbf{A 2}^{2+}$ stations upon electromagnetic stimuli. It was estimated that $\sim 10 \%$ optically excited switching state energy was used for the ring motion with a mechanical power generated at $3 \times 10^{-17} \mathrm{~W} /$ molecule. These molecules can be driven at a frequency of $\sim 1 \mathrm{kHz}$ and are stable for at least $10^{3}$ cycles in solution at ambient temperature [41].

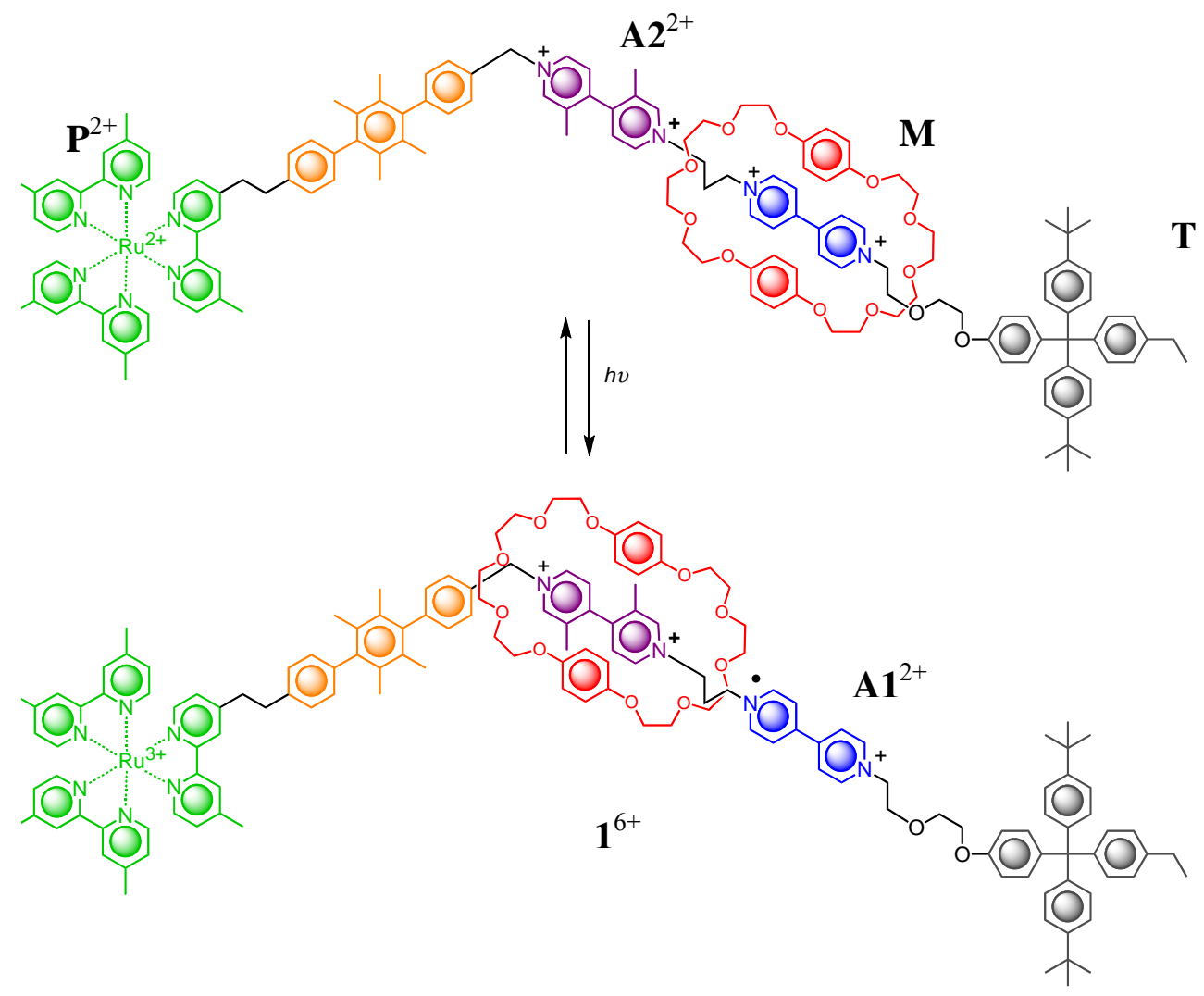

Fig. 2. Structural formula of a light-driven molecular abacus $\mathbf{1}^{6+}$ [41]. Copyright (2006) CSIRO Publishing.

Figure 3 shows a light-driven catenane $2^{2+}$ [42]. The $\mathrm{Ru}(\mathrm{II})$-complexing catenane consists of a 42-membered ring (M42) which contains a 2,2'-bipyridine (bipy) ligand, interlocked with a 63-membered ring (M63) which contains two 1,10- phenanthroline (phen) ligands. The $2^{2+}$ exhibits rotary motion (its mechanical schematic is shown in Fig. 1c) when powered by light and heat. In the starting state of $\mathbf{2}^{2+}$, the interlocked M42 and M63 rings are oriented in such a way that all three bidentate ligands participate in complexation with the $\mathrm{Ru}(\mathrm{II})$ ion to satisfy its octahedral coordination environment. Upon irradiation of $2^{2+}$ with a $250 \mathrm{~W}$ halogen lamp $(\lambda>300 \mathrm{~nm})$ either in $\mathrm{MeCN}$ or $\mathrm{CH}_{2} \mathrm{Cl}_{2}$ in the presence of tetraethylammonium chloride, the photochemical process leads to population of the metal-to-ligand charge-transfer triplet excited state and thus the formation of the ligand-field state, which causes decoordination of the M42 ring from the $\mathrm{Ru}(\mathrm{II})$ center. Two $\mathrm{Cl}^{-}$ions or $\mathrm{MeCN}$ molecules combine with the 
$\mathrm{Ru}(\mathrm{II})$ ion to generate $\mathbf{2}^{\mathbf{\prime}} \mathbf{a}$ or $\mathbf{2}^{\mathbf{\prime}} \mathbf{b}^{2+}$, respectively. Heating of the $\mathbf{2}^{\mathbf{\prime}} \mathbf{a}$ or $\mathbf{2}^{\mathbf{\prime}} \mathbf{b}^{2+}$ solution returns the molecule back into the original isomer $2^{2+}$. The rotary motion has been characterized with absorption and nuclear magnetic resonance (NMR) spectra [42].

Aida et al. have reported the demonstration of light-driven chiral molecular scissors (Fig. 4) [43]. The molecular scissors, like ordinary scissors, consist of three essential components: handles, pivot, and blades. Two phenylene groups were used for the handles, which were strapped by an azobenzene unit through ethylene linkages; $1,1^{\prime}, 3,3^{\prime}$-tetrasubstituted ferrocene was used as the pivot; and two phenyl groups were used as the blades. Upon irradiation with visible and ultraviolet lights, azobenzene experiences photoisomerization and expands and contracts reversibly. The photoisomerization serves as the driving force to manipulate the handles. As the two cyclopentadienyl rings parallel to each other rotate freely, the motion in the handles was transferred into the blades through the pivot. The experimental characterizations of the two-state motion induced by the photoisomerization of the azobenzene unit were carried out with NMR and circular dichroism spectroscopy. Further density functional theory (DFT) calculation indicated that the blades are closed when the connecting azobenzene unit adopts a trans configuration. In this configuration the azobenzene expands, moving the two phenylene groups in the handles apart from each other, simultaneously bringing the phenyl groups in the blades closer to one another with a bite angle of $9.2^{\circ}$. On the other hand, in the cis configuration, the two phenylene groups in the handles are brought close to each other and the blade phenyl groups are separated with a bite angle of $58.2^{\circ}$.

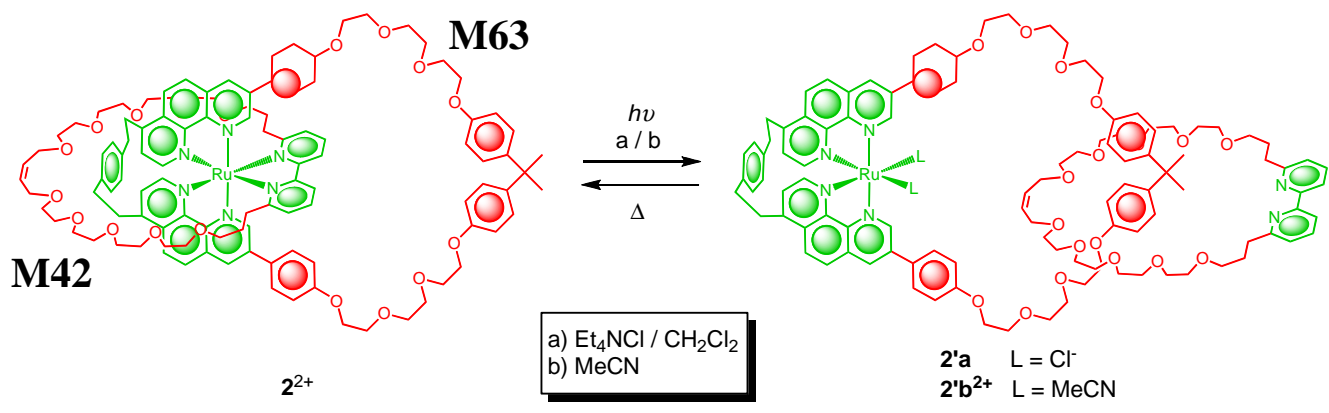

Fig. 3. Structural formula of a light-driven bistable [2]catenane $2^{2+}$ [42]. Copyright (2004) Wiley-VCH.
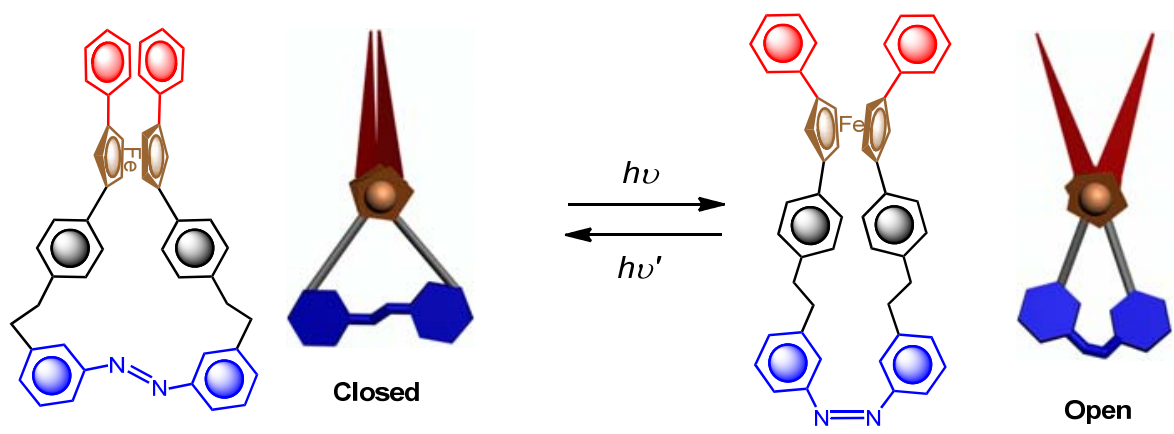

Fig. 4. Structural formula and graphic representative of light-driven chiral molecular scissors based on photoisomerization of azobenzenes. 


\section{ASSEMBLY OF MOLECULAR MACHINES ON SURFACES}

To date, most of the research on molecular machines has been focused on molecular behavior in solution; however, solutions contain a large number of randomly distributed molecules that cannot be addressed individually or expressed coherently, greatly limiting their potential for device functionality [44]. In order to realize the full potential of artificial molecular machine based applications, it is imperative to organize these tiny nanoscale machines at interfaces so that they can function at the nanoscale and be coupled into larger-scale machineries (microscopic, mesoscopic, and macroscopic) [9, 11, 32]. In light of the significance in taking molecular machines from solution to surfaces, this section reviews recent progress in research of molecular machines at surfaces and in devices. The illustrations present a few commonly used approaches for transferring and assembling artificial molecular machines on solid surfaces, i.e., Langmuir-Blodgett (LB) films, self-assembled monolayers (SAMs), and a single molecular machine in matrix (Fig. 5).

(a) Langmuir-Blodgett film

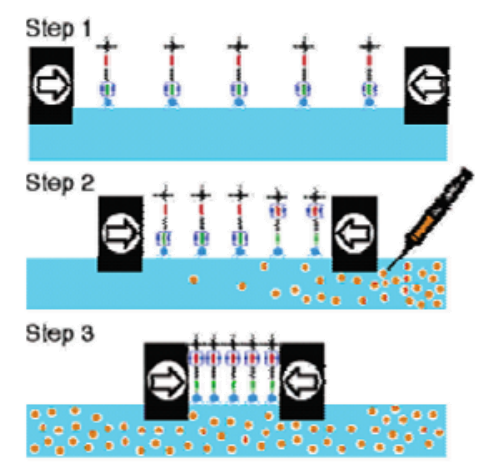

(b) Self-assembled monolayer

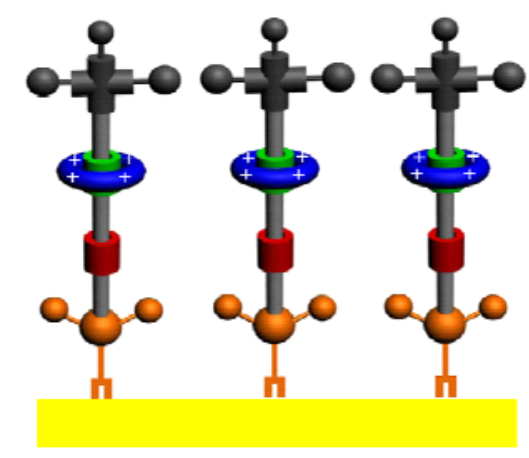

(c) Self-assembled single molecule in matrix

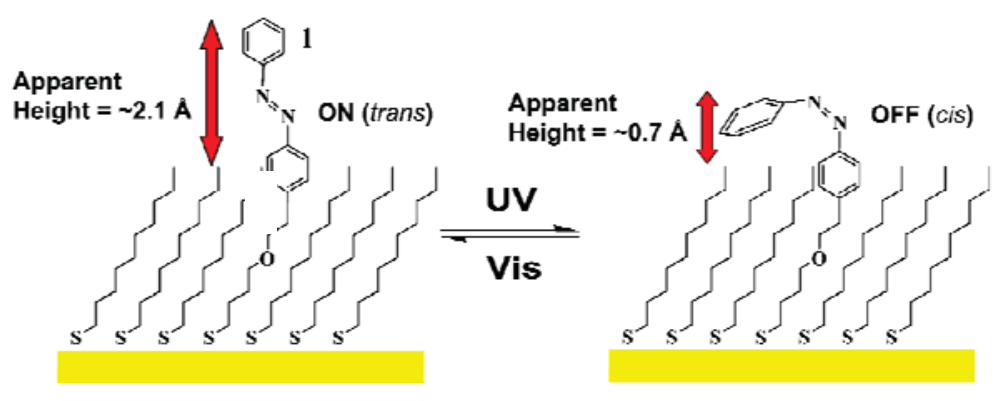

Fig. 5. Approaches for transferring and assembling artificial molecular machines on solid surfaces. (a) Langmuir-Blodgett (LB) films, (b) self-assembled monolayers (SAMs), and (c) a single molecular machine in matrix. Copyright (2008) American Chemical Society.

\subsection{LB thin films}

The LB technique efficiently transfers organized monolayers from the air-water interface onto a solid substrate [45]. This manufacturing approach yields self-organized and oriented amphiphilic molecular machines (Fig. 5a) [8, 46]. In addition, the molecular machines in LB 
films are considered to be in a "liquid crystalline-like" environment, which should help to maintain their mechanical switching properties. The combination of spatial control of superstructure in the solid state and switching behavior in solution state makes the LB technique an ideal one for establishing an interface between amphiphilic, molecular machines and solid supports.

\subsection{SAMs}

SAMs are molecular assemblies that are formed spontaneously by the immersion of an appropriate substrate into a solution of the coating molecule [47]. The self-assembling method can provide highly ordered films without the need for complex equipment. Stoddart and colleagues synthesized bistable rotaxanes with disulfide-based anchoring groups in order to allow self-assembly of rotaxanes onto Au surfaces (Fig. 5b). The switching property of rotaxane SAMs have been observed in multiple experiments involving chemical and electrochemical stimuli [8, 48-54].

\subsection{Single Molecule in Matrix}

The local environment of a single molecular machine or assembly is important to its mechanical properties and it is critical for the interface between the molecule and the microscopic or macroscopic world to be strictly controlled for device applications [55-58]. Weiss and colleagues designed an azobenzene-functionalized molecule, 4-[2-(4-phenylazophenyl)-ethoxy]-butane-1-thiol, to chemisorb within domains of decanethiolate SAMs on $\mathrm{Au}$ (111) substrates through co-assembly (Fig. 5c) [59]. This configuration minimizes conformational changes around the Au-sulfur bond, and gives stability through favorable intermolecular interactions within the monolayer. It also reduces steric constraints as well as electronic coupling between the excited-state orbitals of the molecule and the Au substrate. The molecular tethering scheme allows the azobenzene moiety to protrude from the matrix, which minimizes electronic coupling as well as steric hindrance. Hence, surface quenching and nonphotoinduced switching effects could be limited, and single molecules could be reversibly switched between ON and OFF states by photoirradiation (Fig. 5c) [59].

\section{CHARACTERIZATIONS OF MOLECULAR MACHINES ON SURFACES}

For molecular-machine-based devices to work properly, it is imperative that their dynamic mechanical properties are preserved on the solid-state surfaces [32]. Therefore, it becomes important to be able to characterize the dynamics of molecular machines on surfaces, either in assembly or at the molecular level. Surface confined artificial molecular machines and assemblies can be characterized with many techniques, such as cyclic voltammetry (CV) [6062], X-ray absorption spectroscopy (XAS) [63], X-ray photoemission spectroscopy (XPS) [8, 63], Raman spectroscopy [64], and scanning tunneling microscopy (STM) [59]. In this section, we choose to highlight and discuss a few of these techniques in detail.

$\mathrm{CV}$ is a potentiodynamic electrochemical measurement that is generally used to study the electrochemical properties of an analyte in solution. Typically, a three-electrode setup consisting of a reference electrode, a working electrode, and a counter electrode is used. The electrode potential, measured between reference electrode and working electrode, cycles linearly with time, and the resultant current is measured between working electrode and counter electrode. Finally, a voltage v.s. current curve is generated to ultimately characterize the solution. Stoddart and colleagues have extended CV to investigate the redox behavior of SAMs of bistable rotaxane and dumbbell compound on Au substrates [54]. In the rotaxane, a cyclobis (paraquat-p-phenylene) $\left(\mathrm{CBPQT}^{4+}\right)$ ring can be actively shuttled between the trathiafulvalene (TTF) unit and the 1,5-dioxynaphthalene (DNP) unit during the redox 
process. In the investigation of the TTF-centered oxidation processes, three oxidation peaks were observed for rotaxane at $+310,+490$, and $+630 \mathrm{mV}$ in an intensity ratio of 1:9:10, respectively. By comparing the $\mathrm{CV}$ between rotaxanes and dumbbells, the authors were able to illustrate the presence of two unequally populated (ca. 1:9) translational isomers: the minor one, in which the $\mathrm{CBPQT}^{4+}$ ring encircles the DNP ring system, and the major translational isomer, in which the $\mathrm{CBPQT}^{4+}$ ring encircles the TTF unit. In addition, the CV data of these SAMs displayed a linear increase in current with scan rate, which verifies the presence of a surface-confined redox system. In addition, through the integration of the current with respect to the time associated with each peak, the authors have found the surface coverage of rotaxanes to be 0.25 molecule $/ \mathrm{nm}^{2}$.

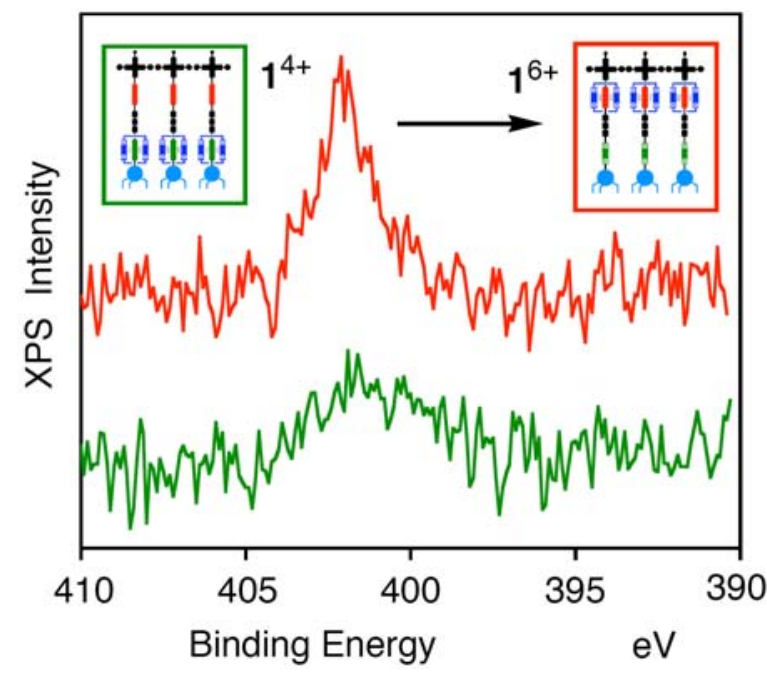

Fig. 6. XPS characterization of the ring motions of rotaxanes on surfaces. The nitrogen intensity is significantly higher in the switched state (shown in red) as compared with that measured in the starting state (shown in green) [8]. Copyright (2004) American Chemical Society.

Vance and colleagues presented clear evidence for the formation of SAMs of rotaxanes on $\mathrm{Au}$ (111) based on XAS and XPS measurements [63]. The SAMs were prepared from an anthracene-stoppered thiol thread, a pseudorotaxane obtained by mixing the thiol with dibenzo-24-crown-8 and a disulfide-containing the [3] rotaxane. XAS and XPS measurements demonstrated that the form of the monolayer precursor dictated the outcome of surface adsorption. Furthermore, using XPS, Huang and colleagues demonstrated, for the first time, that amphiphilic, bistable [2] rotaxanes are mechanically switchable in closely-packed LB films mounted on solid substrates (Fig. 6) [8]. It is well known that, in XPS, the photoemission intensity of each element depends on the depth at which the photoelectron is emitted, with intensity attenuating exponentially with increasing depth. Molecular switching was monitored by using XPS to track nitrogen, which is present only in the rotaxane's mechanically active part: the ring component (inset in Fig. 6). Figure 6 indicates that the density of nitrogen is significantly higher in the switched state (when the molecules are oxidized, shown in red) than in the starting state (shown in green).

Raman spectroscopy, relying on inelastic or Raman scattering of monochromatic light with molecules, is a spectroscopic technique used to study vibrational, rotational, and other low-frequency modes of molecules or their functional groups [65]. The Raman signal of a certain functional group can serve as a fingerprint for the molecule. Although Raman scattering is very weak, only $\sim 10^{-6}$ of the incident light intensity scatters inelastically, when 
the monochromatic excitation matches the electronic transitions in the molecules, resonance Raman occurs and boosts the signal significantly ( $\sim 10^{6}$ times). Flood and collaborators have taken advantage of resonance Raman spectroscopy (RRS) to study the threading behavior of TTF and $\mathrm{CBPQT}^{4+}$ (Fig. 7a) [64]. UV-Vis-NIR spectroscopy reveals an absorption peak at about $865 \mathrm{~nm}$ (Fig. 7b), which is attributed to the TTF to CBPQT ${ }^{4+}$ charge-transfer (CT) transition. An excitation wavelength of $\lambda_{\text {exc }}=785 \mathrm{~nm}$ was used to excite the higher energy side of the CT transition and give a strongly enhanced Raman signal. In the experiments, the TTF was titrated into $0.2 \mathrm{mM} \mathrm{CBPQT}^{4+}$ solution $(\mathrm{MeCN})$ using a tip-drying method. As expected, the $865 \mathrm{~nm} \mathrm{CT}$ absorption band grew during the course of the titration (Fig. 7b). Initially ( 0 equiv TTF), the Raman spectrum was dominated by solvent scattering with only a small $\mathrm{CBPQT}^{4+}$ feature observed at $1650 \mathrm{~cm}^{-1}$. During the titration process, the $\mathrm{CBPQT}^{4+}$-based bands of the complex shifted to $1643 \mathrm{~cm}^{-1}$, while two other $\mathrm{CBPQT}^{4+}$-based bands at 1297 and $1248 \mathrm{~cm}^{-1}$ emerged. All three bands grew with the TTF-based band at $1496 \mathrm{~cm}^{-1}$ throughout the titration process.

(a)

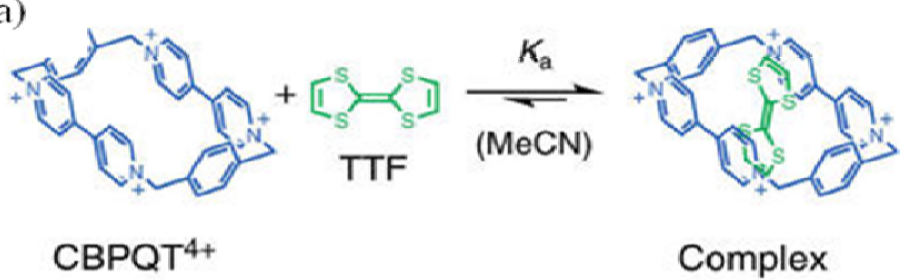

(b)
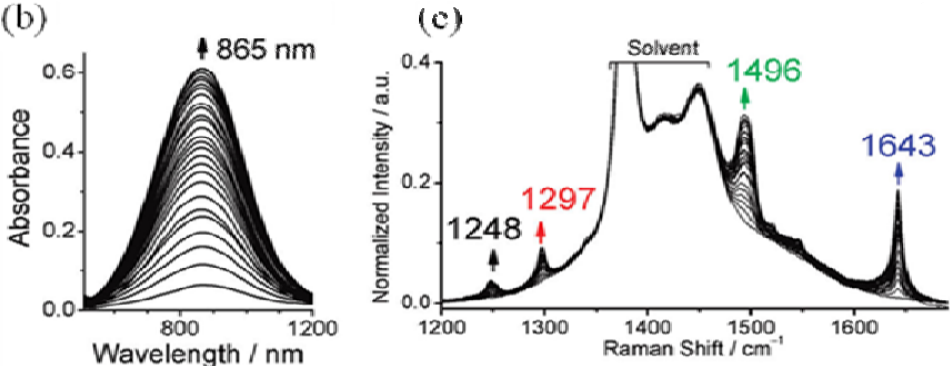

Fig. 7. RRS characterization of the threading behavior of TTF and $\mathrm{CBPQT}^{4+}$. (a) Model complex characterized herein between TTF and CBPQT ${ }^{4+}$. Titration of TTF into $200 \mu \mathrm{M} \mathrm{CBPQT}^{4+}(\mathrm{MeCN})$ as characterized by (b) UV-Vis-NIR spectroscopy and (c) RRS ( $\lambda$ exc $=785 \mathrm{~nm})$ spectroscopy [64]. Copyright (2009) American Chemical Society.

Very recently, Flood and collaborators have moved forward to demonstrate that Raman signals from the threading behavior of TTF and $\mathrm{CBPQT}^{4+}$ can be further enhanced by combining RRS and surface-enhanced Raman spectroscopy (SERS), i.e., surface-enhanced resonance Raman spectroscopy (SERRS) [66]. SERS has attracted great interest for detecting and identifying molecular structures with single molecule sensitivity $[65,67,68]$. Dramatically enhanced local electromagnetic fields associated with excitation of the localized surface plasmon resonances (LSPRs) are the primary mechanism behind the signal enhancement, and thus metal nanostructures are commonly used as SERS substrates [65]. In SERS, when the excitation wavelength overlaps with the LSPRs, the Raman scattering efficiency of a molecular system can be increased by multiple orders of magnitude, which makes Raman signals from SAMs of molecules detectable on metal surfaces. By engineering the shape and size of the metal particles to tune their LSPRs, it is possible to take advantage of both RRS and SERS to achieve an enhancement in Raman signal of up to $\sim 10^{14}$. Such an 
enhancement makes SERRS sensitive enough to monitor the motions of SAMs of molecular machines on surfaces.

STM is a powerful instrument for imaging surfaces at the atomic level with a resolution of $\sim 0.1 \mathrm{~nm}$ or smaller [58]. Recent progress in STM imaging has allowed the visualization of arrangements, orientations, and even inner structures of molecules confined to surfaces. Shigekawa and colleagues have constructed a molecular abacus based on polyrotaxane, consisting of $\alpha$-cyclodextrin ( $\alpha-\mathrm{CD})$ rings and a polyethylene glycol (PEG) chain [69]. Through STM, single $\alpha$-CD rings threaded on the PEG chain were detected and reversibly manipulated with the probe of STM. Weiss and colleagues used STM to study the coassembly of an azobenzene-functionalized molecule, 4-[2-(4-phenylazo-phenyl)-ethoxy]-butane-1-thiol, and decanethiolate on a $\mathrm{Au}$ (111) substrate (Fig. 5c) [59]. As shown in Fig. 5c, without illumination, the azobenzene was in the trans state and imaged as a $2.1 \pm 0.3 \AA$ apparent protrusion from the matrix. The molecules were then exposed to UV light $(\sim 365 \mathrm{~nm})$ and imaged with STM at increasing illumination times, shown in Fig. 8. Upon continuous UV illumination, the molecules switched to the cis state, and were characterized by an apparent height reduction of $\sim 1.4 \AA$ (Fig. 5c), with an increasing number of molecules switched at longer UV illumination times (shown in Fig. 8). After 160 min of UV illumination, more than $90 \%$ of the azobenzene-functionalized molecules isomerized from trans to cis. Subsequent illumination with visible light $\left(\sim 450 \mathrm{~nm}, \sim 6 \mathrm{~mW} / \mathrm{cm}^{2}\right)$ for 30 min switched nearly $50 \%$ of the molecules back to the trans state (Fig. 8F) [59].
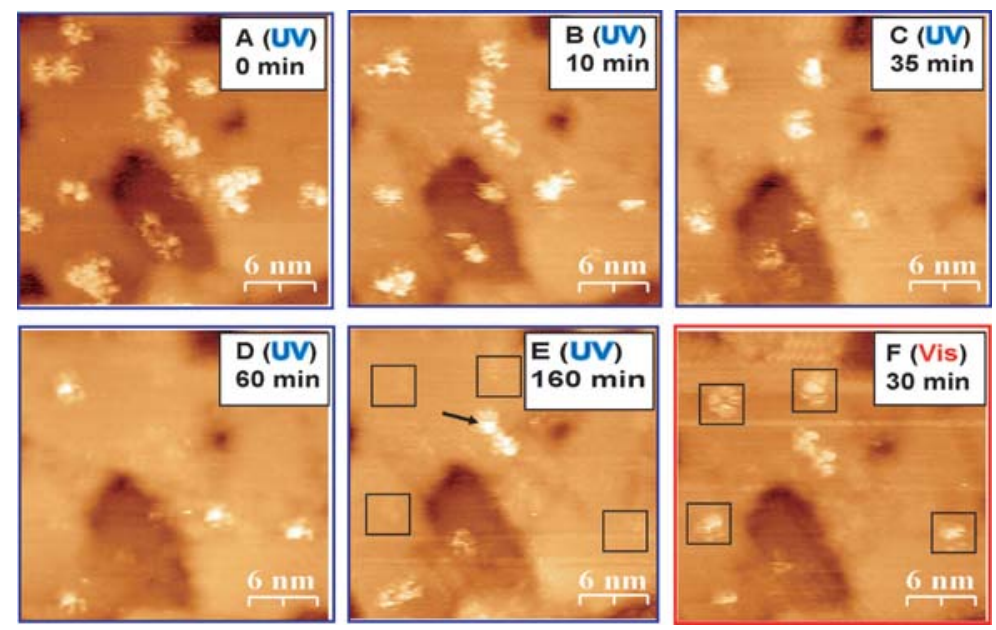

Fig. 8. The STM images revealing the azobenzene-functionalized molecules photoswitching from trans to cis and back, respectively. The isomerization is observed as an apparent height change in STM images. Imaging conditions: $V_{\text {sample }}=$ $1 \mathrm{~V} ; I_{\text {tunnel }}=2 \mathrm{pA}$ [59]. Copyright (2008) American Chemical Society.

\section{APPLICATIONS}

The controllable and reversible molecular-level motions in artificial molecular machines enable important applications across a variety of fields. With the recent advancements in molecular synthesis, characterization, and assembly, light-driven molecular machines have been demonstrated to work as functional elements in molecular electronics, NEMS, nanophotonics, and nanomedicine [3]. A few examples are presented in this section. 


\subsection{NEMS}

Inspired by simple, biological molecular machines in nature, researchers have been utilizing the controllable mechanical motions of artificial molecular machines to develop NEMS that might be able to operate with a similar elegance, efficiency, and complexity as biological motors do within the human body. Although this remains a lofty goal, some impressive progress has been made along this direction in recent years.

As materials for actuation, rotaxanes have many advantages over biological molecular motors and other conventional materials: (1) they can generate strains up to $42 \%$, which are impressive when compared to the strains generated by the gold-standard actuation materials - piezoelectric materials - typically $0.1-0.2 \%$; (2) they have a high force density, e.g., a bistable rotaxane can generate $\sim 100 \mathrm{pN}$ force, while a kinesin biomotor, which is much larger than a bistable rotaxane in size, can only generate $6 \mathrm{pN}$; (3) they can undergo controlled mechanical motion for a variety of external stimuli, while traditional actuation materials and biomotors must rely on a single stimulus; (4) they can be customized and optimized, therefore conferring the flexibility necessary for a multitude of engineering applications; (5) they can survive in a wide range of temperatures $\left(-30-100^{\circ} \mathrm{C}\right)$ and $\mathrm{pH}$ values $(4-10)$, while biomotors are restricted to physiological conditions $\left(\mathrm{T}: \sim 37^{\circ} \mathrm{C}, \mathrm{pH}: \sim 7\right)$ [53]. Therefore, although biomotors are near perfect actuators in human bodies, artificial molecular machines such as bistable rotaxanes are more suitable for the development of active nanostructure-based engineering applications (Table 1).

Table 1. Comparison between biological molecular motors and bistable rotaxanes.

\begin{tabular}{|l|l|l|}
\hline & \multicolumn{1}{|c|}{ Biological Molecular Motors } & \multicolumn{1}{c|}{ Bistable Rotaxanes } \\
\hline $\begin{array}{l}\text { Surviving } \\
\text { Condition }\end{array}$ & $\begin{array}{l}\text { Physiological conditions only } \\
\left(\text { e.g., T: } \sim 37^{\circ} \mathrm{C}, \mathrm{pH}: \sim 7\right)\end{array}$ & $\mathrm{T}:-30-+100^{\circ} \mathrm{C}, \mathrm{pH}: 4-10$ \\
\hline Flexibility & $\begin{array}{l}\text { Difficult to modify structures and } \\
\text { functions }\end{array}$ & Easy to modify \\
\hline Stimuli & Chemical only & Chemical, electricity, or light \\
\hline Frequency & $\sim 1 \mathrm{~Hz}$ & $\mathrm{Up} \mathrm{to} 100 \mathrm{GHz}$ (theoretically) \\
\hline Size & $30-100 \mathrm{~nm}^{3}$ & $8 \mathrm{~nm}^{3}$ \\
\hline Force & $\sim 6 \mathrm{pN}($ Kinesin motor $)$ & $40-100 \mathrm{pN}$ \\
\hline
\end{tabular}

Huang and colleagues have proven a molecular-machine-based mechanical actuator by utilizing a hybrid top-down/bottom-up manufacturing approach [49, 50, 52]. Here the concept of a bistable [2]rotaxane was extended into a doubly bistable palindromic [3] rotaxane to develop a molecular muscle. As shown in Fig. 9a, the palindromic bistable [3] rotaxane is composed of a symmetrical dumbbell component with two rings interlocked onto the dumbbell. The distance between two rings contracts and extends upon oxidation and reduction. Each ring carries a disulfide tether to allow the self-assembly of the rotaxane onto $\mathrm{Au}$ surface. These molecular muscles, when self-assembled on microcantilever beams $(500 \times 100 \times 1 \mu \mathrm{m})$, are capable of bending and stretching the beams during a redox process by stimulated by chemical or electrochemical methods. Figure $9 \mathrm{~b}$ shows the time-dependent operation of the rotaxane-assembled microcantilever. In this electrochemical method, the redox state was changed by applying a desired potential to the cantilever using a potentiostat, and the deflection magnitude was monitored by variations in a reflected laser beam's position on a photodiode. The potential steps were alternated in order to initiate oxidation and reduction, and the deflections were measured as a function of time (Fig. 9b). The alternating 
bending, down and up, of the microcantilever corresponds to the alternating oxidation and reduction of the surface-coated muscle molecules, respectively.

Conversely, microcantilever beams that were coated with a redox-active, but mechanically inert control compound did not display the same bending characteristics. Heat, photo-thermal effects, and $\mathrm{pH}$ variation, as potential mechanisms for the observed curvature in the microcantilever beams, were precluded by a series of control experiments. Moreover, using elementary beam theory and analysis, it was shown that these observations support the notion that cumulative nanoscale movements within surface-bound molecular muscles can be harnessed to perform larger-scale mechanical work.
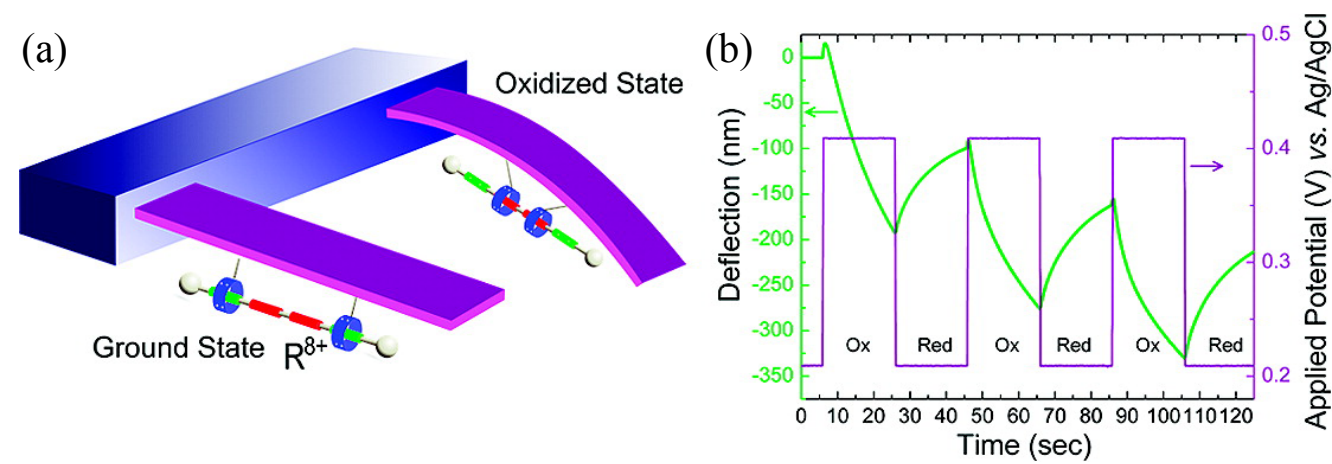

Fig. 9. A mechanical actuator driven by molecular machines. (a) Schematic showing how the reversible oxidation and reduction of the palindromic bistable [3] rotaxane $\mathbf{R}^{8+}$ produced the deflection of microcantilever, (b) experimental data showing the time-dependent operation of the microcantilever coated with $\mathbf{R}^{\mathbf{8}^{+}}$driven by electrochemical stimulus. Copyright (2009) American Chemical Society.

(a)

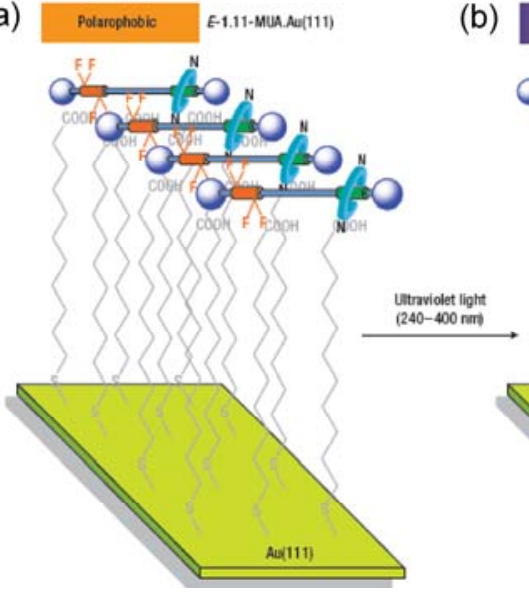

(b)

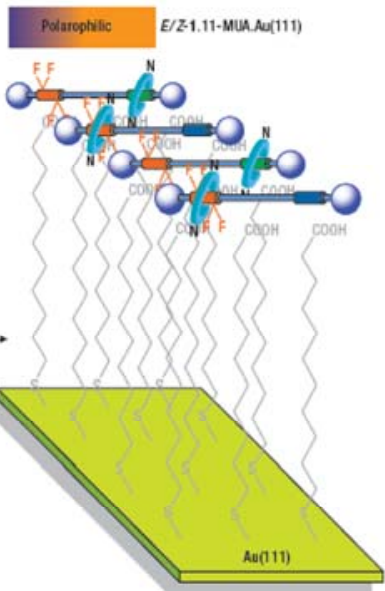

(c)

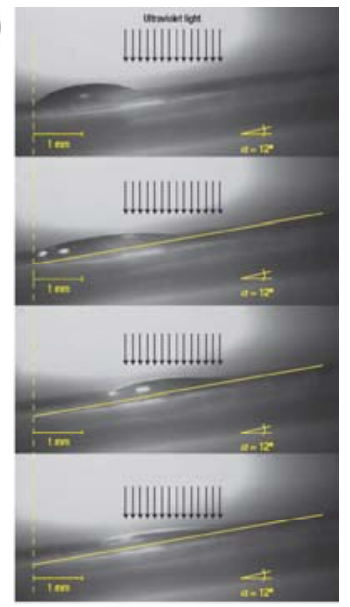

Fig. 10. Macroscopic transport of liquids by surface-bound molecular machines. (a\&b) Schematic drawing of light-switchable rotaxanes physisorbed onto a SAM of 11-MUA on Au (111) surface, (c) illumination with $240-400 \mathrm{~nm}$ light causes the rings shuttle to the fluoroalkane units, leaving a more hydrophilic surface and driving the droplet uphill. Copyright (2005) Nature Publishing Group.

In another application, Berna and colleagues demonstrated the use of a molecularmachine-based photoresponsive surface to control macroscopic liquid transport [70]. In this 
work, a SAM of 11-mercapoundeconoic acid (11-MUA) was assembled onto a gold surface and photoactive rotaxanes were physisorbed onto the SAM with the dumbbell components parallel to the Au surface (Fig. 10). This rotaxane was comprised of a ring and a dumbbell with a fluoroalkane station and a photoresponsive fumaramide station. Photoisomerization results in a transformation of the fumaramide into maleamide, which has a much lower affinity for the ring component. Thus, irradiation of light reduces the binding affinity of the photoresponsive station to the ring drastically, and the equilibrium position of the ring is in favor of the fluoroalkane station. The ring movement can be used to expose or conceal the fluoroalkane station, thus changing the surface energy. When small drops of low-volatility liquids (e.g., $\mathrm{CH}_{2} \mathrm{I}_{2}$ ) were deposited onto the molecular-machine-based photoresponsive surface, the collective operation of the rotaxane monolayer was sufficient to power the movement of a microlitre droplet up a twelve degree incline (Fig. 10c). The experiment showed nearly $\sim 50 \%$ efficiency—approximately $50 \%$ of the light energy absorbed by rotaxane was used to overcome the effect of gravity.

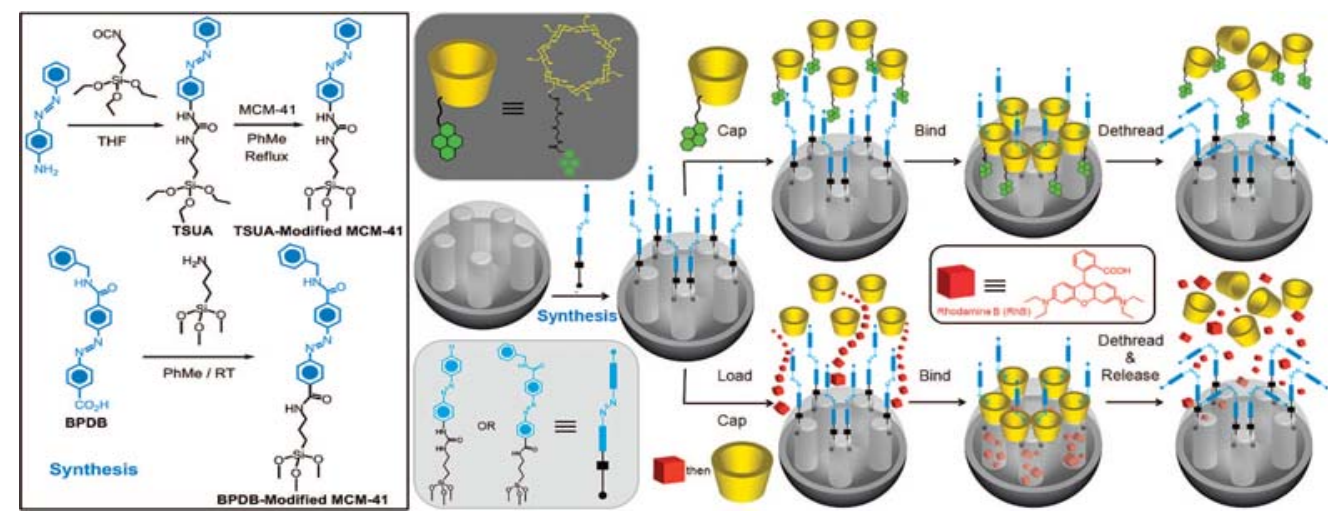

Fig. 11. Artificial molecular machines work as nanovalves for drug delivery. Py- $\beta$ $\mathrm{CD}$ or $\beta-\mathrm{CD}$ threads onto the trans-AB stalks to seal the nanopores. Upon irradiation $(351 \mathrm{~nm})$, the isomerization of trans-to-cis $\mathrm{AB}$ units leads to the dissociation of Py- $\beta-C D$ or $\beta-C D$ rings from the stalks, thus opening the gates to the nanopores and releasing the cargo. Copyright (2009) American Chemical Society.

\subsection{Nanomedicine}

Nanomechanical systems that are designed to trap and release cargoes from carriers in response to a stimulus are potentially useful in medical applications, mainly precise drug targeting and delivery [71]. Nanomaterials suitable for this type of operation must consist of an appropriate container with stimulus-activated nanovalves. Many research groups have demonstrated that artificial molecular machines with well-controlled movable components can act as efficient nanovalves for precise drug delivery once assembled properly onto the appropriate containers [71-74].

Prototypes of these delivery systems have been realized with photoactivated moving parts based on the photoisomerization of azobenzene derivatives that have been integrated with mesoporous silica. The decrease in the size accompanying the transition from trans to cis isomers in azobenzene molecules attached to pore interiors has been used to regulate the transport of molecules [75]. In another example, Stoddart and colleagues reported an approach that takes advantage of the difference in binding affinity between $\beta-\mathrm{CD}$ and trans- 
azobenzene (high) and $\beta-\mathrm{CD}$ and cis-azobenzene (low) to control the release of cargo molecules from mechanized silica nanoparticles (Fig. 11) [76]. The functionality was demonstrated on two MCM-41 nanoparticles modified with azobenzene derivatives (AB). The $\mathrm{AB}$ were prepared from 4-(3- triethoxysilylpropylureido) azobenzene (TSUA) and (E)-4((4-(benzylcarbamoyl)phenyl)diazenyl) benzoic acid (BPDB) with pyrene-modified $\beta$-CD $(\mathrm{Py}-\beta-\mathrm{CD})$ and $\beta-\mathrm{CD}$ as caps, respectively. For $\mathrm{AB}$, trans is a more stable configuration. In the case of MCM-41 carrying AB-containing stalks, $\beta-C D$ or Py- $\beta-C D$ threads onto the stalks and bind to trans- $\mathrm{AB}$ units, thus sealing the nanopores and stopping release of the cargo. However, upon irradiation of $351 \mathrm{~nm}$ light, $\mathrm{AB}$ isomerizes to the less stable cis configuration, resulting in the dissociation of $\beta-C D$ or Py- $\beta-C D$ from the stalks, which opens the gates to the nanopores and releases the cargo. The fluorescence of Py- $\beta-C D$ was first used to characterize the dissociation of the rings from the nanoparticles. Results from the more water-soluble BPDB-derived stalks on the nanoparticles further confirmed that $\beta-\mathrm{CD}$ rings can prevent the cargo, Rhodamine $\mathrm{B}$, from leaving the MCM-41 nanopores when they bind with the $\mathrm{AB}$ trans units; the rings can also release the cargo once they are dissociated with the $\mathrm{AB}$ cis units as a consequence of the light-promoted isomerization.

\subsection{Nanophotonics}

Research in nanophotonics investigates and utilizes the interaction of light with nanostructures [77]. The phenomena that occur when light interacts with structures at the nanoscale are interesting both for scientific purposes and the development of novel photonic devices [78-80]. Artificial molecular machines can provide controlled mechanical motions at the nanometer scale: a property that could vastly impact emerging functional elements for nanophotonics. Molecular-machine-based functional photonic devices have targeted many important applications such as light harvesting, display, and nanoscale optical integrated circuits $[81,82]$. In this subsection, we give a few examples of the applications of artificial molecular machines in the field of nanophotonics.

\subsubsection{Light harvesting}

Energy presents perhaps the most significant challenge of the 21st century. The electromagnetic radiation from the sun provides quantities of energy far exceeding human needs, and could become the most significant source of renewable energy for the development of our society if it can be efficiently harvested. Among many different methods to capture sunlight, photovoltaics (PV) have emerged as one of the most prominent ways to convert sunlight directly into photocurrent that can power anything that runs on electricity. Stoddart and colleagues have devised a sunlight-powered artificial molecular machine; it uses a switching element based on noncovalent interactions within recognition units to execute mechanical movement. A light-harvesting unit utilizes the sun's radiation to control the competitive interactions between the recognition sites [83]. As shown in Fig. 12, a molecular triad 11, composed of (i) an electron-donating TTF unit, (ii) a chromophoric porphyrin unit, and (iii) an electron-accepting $\mathrm{C}_{60}$ unit, has been used to harness light and convert it into electrical energy. The electrical energy was then utilized as a nanoscale power supply to drive the dethreading of the BHEEN $\subset \mathrm{CBPQT}^{4+}$ pseudorotaxane (Fig. 12). In the presence of 413 $\mathrm{nm}$ light, $6.7 \%$ of the $0.37 \mathrm{mM}$ pseudorotaxane in MeCN could be dethreaded over $2900 \mathrm{~s}$. 


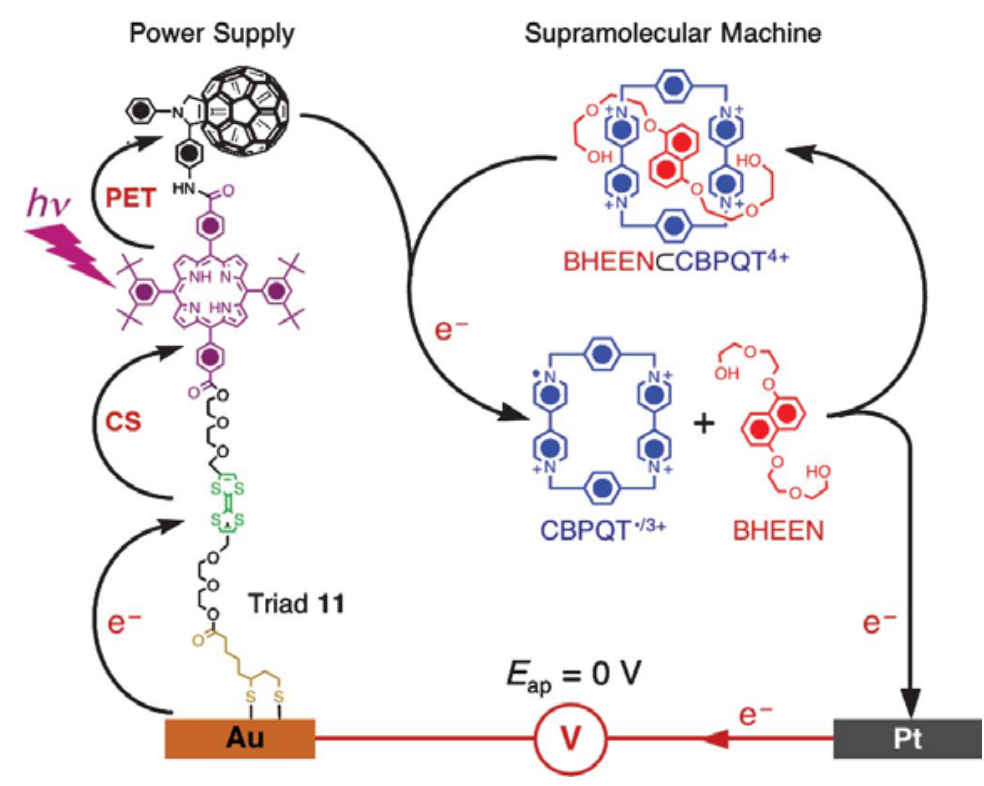

Fig. 12. Powering of a supramolecular machine BHEEN $\subset \mathrm{CBPQT}^{4+}$ with photocurrent generated by the molecular triad 11 [83]. Copyright (2005) Wiley$\mathrm{VCH}$.

\subsubsection{Full-color electronic paper display}

Electronic paper displays (E-PADs) are an ultrathin and flexible electronic display that combines the viewing characteristics of conventional printed paper with the ability to electronically manipulate the displayed information; these EPADs attract great interest for applications in wearable computer screens and electronic newspapers [84]. Although black/white E-PAD prototypes have been demonstrated $[85,86]$, the full-color display is the ultimate goal. The existing technologies for producing full-color E-PADs have several shortcomings. For example, high complexity and cost arise from the use of different lightemitting materials to provide red (R), green $(\mathrm{G})$, and blue (B) sub-pixels. Current pixel components based on electrowetting manipulation of microscale liquid droplets have limited resolution [86].

The development of novel organic materials, such as mechanically interlocked molecular machines, could offer an alternative way to construct thin, flexible, and light-weight E-PADs. Figure 13 illustrates the concept of electrochemically controllable RGB catenane display [87]. In the [2]catenane, changing the location of the $\mathrm{CBPQT}^{4+}$ ring between the three $\pi$-electron rich stations generates three different colors $(R, G, B)$ by varying the $C T$ interactions between the $\mathrm{CBPQT}^{4+}$ ring and the three distinct stations. As a preliminary step, Stoddart et al. have demonstrated the electrochromism of two-station [2]catenanes in a polymer gel matrix (polymethylmethacrylate gel) [88]; they used CV measurement to monitor the kinetics of the electrochromism. In the neutral state, the $\mathrm{CBPQT}^{4+}$ ring resides preferentially on the TTF site (ground state), and the polymer matrix shows green color. The oxidation of the TTF unit is accompanied by a rapidly driven shuttling of the $\mathrm{CBPQT}^{4+}$ ring to the DNP site and a color change of the polymer matrix from green to red/purple. Upon reduction, $\mathrm{CBPQT}^{4+}$ relaxes from the metastable state and returns to its charge-neutral state (green color). Recent theoretical studies from Stoddart's group predicted that RGB colors should be obtained from the inclusion complexes formed between $\mathrm{CBPQT}^{4+}$ and DNP-TEG, TTF-TEG and DFBZ- 
TEG, respectively $[89,90]$. Experimental realization of the three-station [2]catenanes for RGB colors is currently underway.

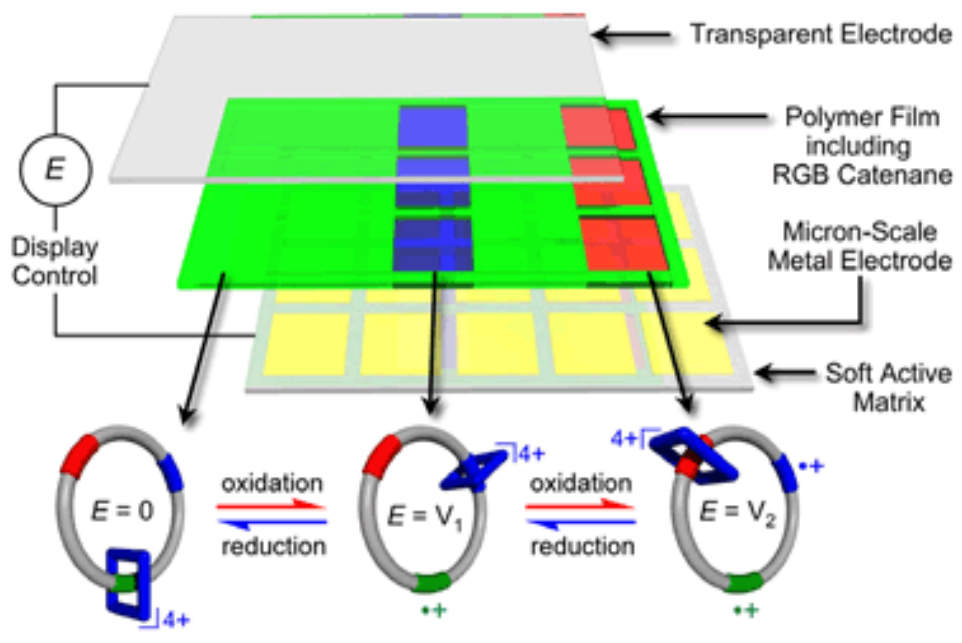

Fig. 13. The proposed design for electronic paper display (E-PAD) based on the electrochromism of electrochemically-controllable three-station [2]catenanes. The control voltage is applied between the transparent electrode and the soft active matrix to manipulate the three-station [2]catenanes which are capable of exhibiting RGB colors in response to the applied voltage. The RGB colors originate from the CT absorption bands between the $\mathrm{CBPQT}^{4+}$ and the three stations on the macrocyclic polyethers [87]. Copyright (2008) IOP Publishing Ltd.

The proposed method using novel RGB tristable three-station [2]catenane has many advantages when compared to the existing technologies for full-color E-PADs. First of all, the proposed method consumes much less power: while the existing technologies require at least a $15 \mathrm{~V}$ driving voltage $[86,91,92]$, control of the tristable [2]catenane, and thus the catenanebased E-PADs, requires only $0.5-2 \mathrm{~V}$. Second, each pixel only needs a single basic cell instead of three to generate R, G, and B colors. This feature would dramatically reduce the complexity and the cost of the resulting device. Third, since the proposed E-PAD is based on molecular behavior, it can provide up to nanometer resolution. Finally, it has higher dynamic range (100\% switching) than existing technologies ( $75 \%$ switching). We envision that if realized, the RGB catenanes will pave the way for the cost-effective production of highfrequency, high-resolution, energy-efficient, full-color E-PADs.

\subsubsection{Molecular plasmonic switches}

Surface-plasmon-based nanophotonics [81, 93], or "plasmonics," can potentially guide and manipulate light at subwavelength scales and thereby allow the development of nanophotonic integrated circuits $[94,95]$. Although major breakthroughs have been made in plasmonic waveguides [96-100], couplers [101, 102], and lenses [103, 104], a limited amount of research has been conducted on active plasmonic components, such as switches and modulators $[105,106]$. Recently, active plasmonic systems that used metal nanoparticle/molecule complexes were demonstrated and shown to have considerable promise as a new class of ultrasmall plasmonic components [107-109]. In these plasmonic systems, the molecules shift the localized surface plasmon resonance (LSPR) of the metal nanostructures by changing the coupling between surface plasmon resonances and molecular 
resonances [110-115]. To date, the LSPR shifts in most of these systems are irreversible processes [110-115]. To realize the potential of molecular-level active plasmonics, a reversible shift of LSPR is needed [107]. Herein, Zheng and colleagues take advantage of the reversible, controllable mechanical motions in molecular machines, such as switchable bistable rotaxanes, to demonstrate a molecular-level plasmonic switch that can be reversibly controlled by molecular machines.
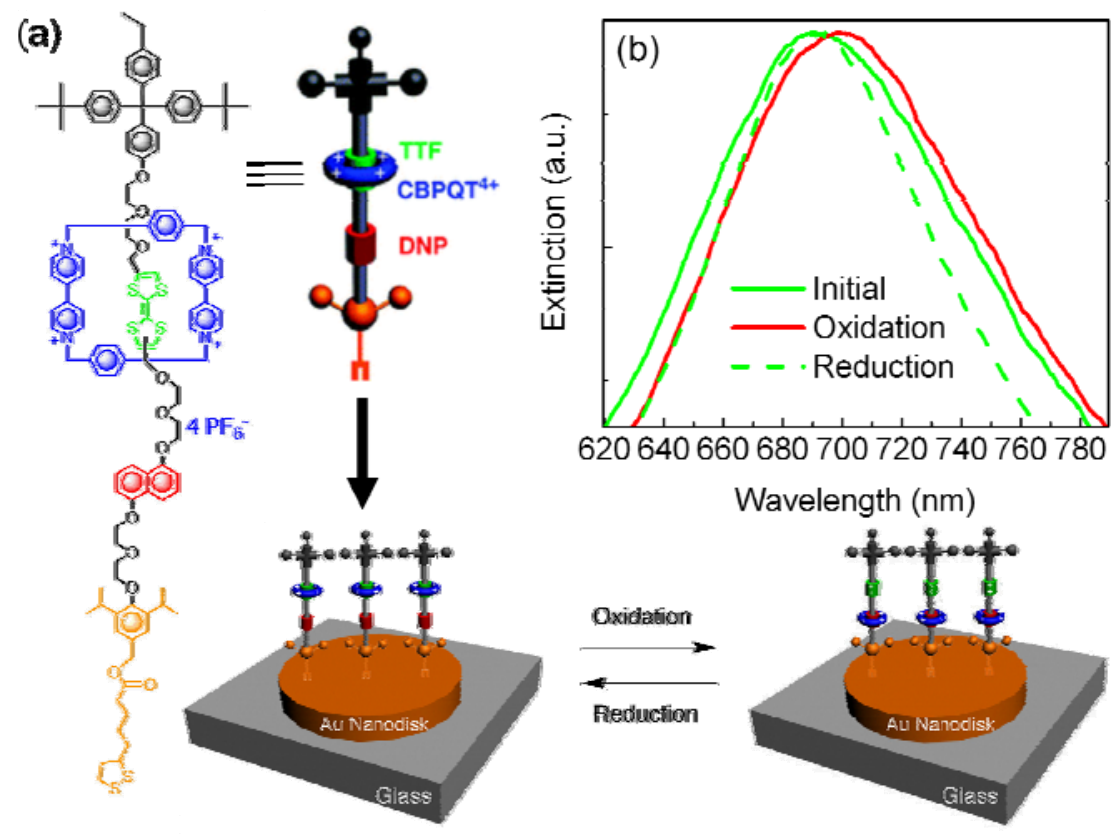

Fig. 14. Molecular plasmonic switches with molecular machines as functional elements. (a) The disulfide functional groups appended to rotaxanes were used to immobilize the molecules onto the $\mathrm{Au}$ nanodisk surfaces as chemically bound monolayers. (b) Extinction spectra recorded from rotaxane-derivatized $\mathrm{Au}$ nanodisks in air with an initial peak wavelength of $690.4 \pm 0.2 \mathrm{~nm}$ during the redox process [48]. Copyright (2009) American Chemical Society.

As shown in Fig. 14a, SAMs of disulfide-tethered bistable rotaxanes were formed on $\mathrm{Au}$ nanodisks by incubating the nanodisks in a solution $(0.1 \mathrm{mM}$ in $\mathrm{MeCN})$ of rotaxanes [48]. The Au nanodisks were fabricated on glass substrates using nanosphere lithography [116]. The extinction spectra for the rotaxane-derivatized Au nanodisks in an oxidation/reduction cycle are shown in Fig. 14b. Oxidation of the rotaxanes caused the LSPR peak to red shift from $690.4 \pm 0.2$ to $699.9 \pm 0.2 \mathrm{~nm}$, while reduction caused the peak to blue shift back to its initial position. The reversible peak shift was reproducible through many oxidation and reduction cycles. Further control experiments and a time-dependent density functional theory (TDDFT)-based microscopic mode support the idea that the reversible switching in the LSPR of Au nanodisk arrays correlates with the chemically-driven mechanical switching observed for surface-bound bistable [2] rotaxane molecules.

The active molecular plasmonic system demonstrated here can be differentiated from existing systems in the following aspects: (i) the active component, bistable rotaxane, is one of a unique class of systems that can deliver controllable, reversible mechanical motions at the molecular level; (ii) the LSPR modulations observed here have been obtained for from a monolayer $(<10 \mathrm{~nm}$ thickness) of molecules, rather than from thin films or polymer matrices 
(>50 nm thickness) [117-120]; and (iii) unlike some molecular plasmonic systems that only function in solution [121-124], the bistable rotaxanes operate after being immobilized onto solid substrates. Furthermore, the structure and properties of the wholly synthetic bistable rotaxanes can be customized and optimized. The flexibility in chemical structure enables the development of various bistable rotaxane-based molecular switches that have prescribed optical properties and a wide range of possibilities for external stimuli, i.e., chemical, electrochemical, or photochemical [3]. The latter two stimuli are particularly promising because (i) the act of switching is simple, (ii) the switching takes place without producing chemical waste, and (iii) the molecular mechanical motions are induced and detected using only electricity or light. It is plausible that the chemically-driven redox process could be replaced with direct electrical or optical stimulation; this development has the potential to establish a technological basis for the production of a new class of molecular-machine-based active plasmonic components for solid-state nanophotonic integrated circuits with the potential for low-energy, nanoscale operations.

\section{CONCLUSIONS}

In this article we reviewed recent advances in light-driven artificial molecular machines. Although the field may still be young, research in the area of light-driven artificial molecular machines has gained momentum in the past decade. Many molecular machines with precisely-tailored structures and functions (e.g., molecular analogs of rotors, gears, switches, and shuttles) have been synthesized. Aided by the development of different surfaceassembling methods and characterization tools, significant efforts have been made to move the research on molecular machines from solution, to surfaces, to devices settings $[125,126]$. With the recent advancements in molecular synthesis, assembly, and characterization, lightdriven molecular machines have been demonstrated to work as functional elements in numerous applications. For example, cumulative nanoscale movements within molecular machines have been harnessed to perform large-scale mechanical work (e.g., driving microcantilever beams and manipulating microliter droplets). Light-driven molecular machines have also been utilized in biomedical applications such as drug delivery. Most recently, the mechanically induced optical switching properties of molecular machines have been utilized in nanophotonic applications, such as light harvesting, electronic paper display, and molecular active plasmonic devices. Just as individual molecules must organize into coherent assemblies for larger-scale work, so must chemists, material scientists, and engineers work together to promote a better fundamental understanding of light-driven molecular machines in surface-bound and solid-state environments. Major challenges in this field remain, such as unidirectional molecular rotors, faster molecular motions over longer time frames, molecular machines with rigid backbones, and bio-compatible, light-driven molecular machines. Despite these challenges, we believe that with concerted, collaborative efforts across various disciplines, multifunctional molecular machinery has promising applications over multiple scales (i.e., nano, micro, meso, and macro) for applications in molecular electronics [19], NEMS [127], nanomedicine [128,129], and nanophotonics [130-133].

\section{Acknowledgments}

This research was supported by the Air Force Office of Scientific Research, the National Science Foundation, and the Penn State Center for Nanoscale Science (MRSEC). Y.B.Z. acknowledges the support of the Founder's Prize and Grant of the American Academy of Mechanics. The authors thank Dr. J. Fraser Stoddart, Dr. Paul S. Weiss, Dr. Lasse Jensen, Dr. Amar H. Flood, Dr. Vincent Crespi, Lei Fang, and Bala Krishna Juluri for helpful discussions. 


\section{References}

[1] J. Howard, "Molecular motors: structural adaptations to cellular functions," Nature 389, 561-567 (1997) [doi:10.1038/39247].

[2] V. Balzani, A. Credi, F. M. Raymo, and J. F. Stoddart, "Artificial molecular machines," Angew. Chem. Int. Ed. 39, 3349-3391 (2000) [doi:10.1002/15213773(20001002)39:19<3348::AID-ANIE3348>3.0.CO;2-X].

[3] V. Balzani, A. Credi, and M. Venturi, Molecular Devices and Machines: Concepts and Perspectives for the NanoWorld, Wiley-VCH, Weinheim (2008).

[4] R. P. Feynman, "There is plenty of room at the bottom," Eng. Sci. 23, 22-36 (1960).

[5] S. Shinkai and O. Manabe, "Photocontrol of ion extraction and ion-transport by photofunctional crown ethers," Top. Curr. Chem. 121, 67-104 (1984) [doi: 10.1007/3540-12821-2_3].

[6] J. E. Green, J. W. Choi, A. Boukai, Y. Bunimovich, E. Johnston-Halperin, E. DeIonno, Y. Luo, B. A. Sheriff, K. Xu, Y. S. Shin, H. R. Tseng, J. F. Stoddart, and J. R. Heath, "A 160-kilobit molecular electronic memory patterned at 10(11) bits per square centimetre," Nature 445, 414-417 (2007) [doi:10.1038/nature05462].

[7] I. Aprahamian, T. Yasuda, T. Ikeda, S. Saha, W. R. Dichtel, K. Isoda, T. Kato, and J. F. Stoddart, "A liquid-crystalline bistable [2]rotaxane," Angew. Chem. Int. Ed. 46, 4675-4679 (2007) [doi:10.1002/anie.200700305].

[8] T. J. Huang, H. R. Tseng, L. Sha, W. X. Lu, B. Brough, A. H. Flood, B. D. Yu, P. C. Celestre, J. P. Chang, J. F. Stoddart, and C. M. Ho, "Mechanical shuttling of linear motor-molecules in condensed phases on solid substrates," Nano Lett. 4, 2065-2071 (2004) [doi:10.1021/n1035099x].

[9] J. J. Davis, G. A. Orlowski, H. Rahman, and P. D. Beer, "Mechanically interlocked and switchable molecules at surfaces," Chem. Commun. 46, 54-63 (2010) [doi:10.1039/b915122b].

[10] J. P. Sauvage and C. D. Buchecker, Molecular Catenanes, Rotaxanes and Knots, Wiley-VCH, Weinheim (1999) [doi:10.1002/9783527613724].

[11] V. Balzani, A. Credi, and M. Venturi, "Molecular machines working on surfaces and at interfaces," Chem. Phys. Chem. 9, 202-220 (2008) [doi:10.1002/cphc.200700528].

[12] G. Berkovic, V. Krongauz, and V. Weiss, "Spiropyrans and spirooxazines for memories and switches," Chem. Rev. 100, 1741-1753 (2000) [doi:10.1021/cr9800715].

[13] J. P. Collin, C. Dietrich-Buchecker, P. Gavina, M. C. Jimenez-Molero, and J. P. Sauvage, "Shuttles and muscles: linear molecular machines based on transition metals," Acc. Chem. Res. 34, 477-487 (2001) [doi:10.1021/ar0001766].

[14] Z. Dominguez, H. Dang, M. J. Strouse, and M. A. Garcia-Garibay, "Molecular "compasses" and "gyroscopes." - III. Dynamics of a phenylene rotor and clathrated benzene in a slipping-gear crystal lattice," J. Am. Chem. Soc. 124, 7719-7727 (2002) [doi:10.1021/ja025753v].

[15] B. L. Feringa, "The art of building small: from molecular switches to molecular motors," J. Org. Chem. 72, 6635-6652 (2007) [doi:10.1021/jo070394d].

[16] B. L. Feringa, Molecular Switches, Wiley-VCH, Weinheim (2001) [doi:10.1002/3527600329].

[17] B. L. Feringa, R. A. van Delden, N. Koumura, and E. M. Geertsema, "Chiroptical molecular switches," Chem. Rev. 100, 1789-1816 (2000) [doi:10.1021/cr9900228].

[18] A. H. Flood, R. J. A. Ramirez, W. Q. Deng, R. P. Muller, W. A. Goddard, and J. F. Stoddart, "Meccano on the nanoscale - a blueprint for making some of the world's tiniest machines," Austral. J. Chem. 57, 301-322 (2004) [doi:10.1071/CH03307].

[19] A. H. Flood, J. F. Stoddart, D. W. Steuerman, and J. R. Heath, "Whence molecular electronics?" Science 306, 2055-2056 (2004) [doi:10.1126/science.1106195]. 
[20] C. E. Godinez, G. Zepeda, and M. A. Garcia-Garibay, "Molecular compasses and gyroscopes. II. Synthesis and characterization of molecular rotors with axially substituted bis[2-(9-triptycyl)ethynyl]arenes," J. Am. Chem. Soc. 124, 4701-4707 (2002) [doi:10.1021/ja012550i].

[21] A. Harada, "Cyclodextrin-based molecular machines," Acc. Chem. Res. 34, 456-464 (2001) [doi:10.1021/ar0001741].

[22] E. R. Kay and D. A. Leigh, "Hydrogen bond-assembled synthetic molecular motors and machines," Top. Curr. Chem. 262, 133-177 (2005) [doi:10.1007/128 011].

[23] T. R. Kelly, H. De Silva, and R. A. Silva, "Unidirectional rotary motion in a molecular system," Nature 401, 150-152 (1999) [doi:10.1038/43639].

[24] K. Oh, K. S. Jeong, and J. S. Moore, "Folding-driven synthesis of oligomers," Nature 414, 889-893 (2001) [doi:10.1038/414889a].

[25] C. A. Schalley, K. Beizai, and F. Vogtle, "On the way to rotaxane-based molecular motors: Studies in molecular mobility and topological chirality," Acc. Chem. Res. 34, 465-476 (2001) [doi:10.1021/ar000179i].

[26] S. Shinkai, M. Ikeda, A. Sugasaki, and M. Takeuchi, "Positive allosteric systems designed on dynamic supramolecular scaffolds: Toward switching and amplification of guest affinity and selectivity," Acc. Chem. Res. 34, 494-503 (2001) [doi:10.1021/ar000177y].

[27] Y. Yokoyama, "Fulgides for memories and switches," Chem. Rev. 100, 1717-1739 (2000) [doi:10.1021/cr980070c].

[28] A. Coskun, D. C. Friedman, H. Li, K. Patel, H. A. Khatib, and J. F. Stoddart, "A lightgated STOP-GO molecular shuttle," J. Am. Chem. Soc. 131, 2493-2495 (2009) [doi:10.1021/ja809225e].

[29] D. H. Qu, G. C. Wang, J. Ren, and H. Tian, "A light-driven rotaxane molecular shuttle with dual fluorescence addresses," Org. Lett. 6, 2085-2088 (2004) [doi:10.1021/o1049605g].

[30] M. M. Pollard, M. K. J. ter Wiel, R. A. van Delden, J. Vicario, N. Koumura, C. R. van den Brom, A. Meetsma, and B. L. Feringa, "Light-driven rotary molecular motors on gold nanoparticles," Chem. Eur. J. 14, 11610-11622 (2008) [doi:10.1002/chem.200800814].

[31] K. Ichimura, S. K. Oh, and M. Nakagawa, "Light-driven motion of liquids on a photoresponsive surface," Science 288, 1624-1626 (2000) [doi:10.1126/science.288.5471.1624].

[32] W. R. Browne and B. L. Feringa, "Light switching of molecules on surfaces," Ann. Rev. Phys. Chem. 60, 407-428 (2009) [doi:10.1146/annurev.physchem.040808.090423].

[33] P. R. Ashton, R. Ballardini, V. Balzani, I. Baxter, A. Credi, M. C. T. Fyfe, M. T. Gandolfi, M. Gomez-Lopez, M. V. Martinez-Diaz, A. Piersanti, N. Spencer, J. F. Stoddart, M. Venturi, A. J. P. White, and D. J. Williams, "Acid-base controllable molecular shuttles," J. Am. Chem. Soc. 120, 11932-11942 (1998) [doi:10.1021/ja982167m].

[34] J. W. Lee, K. P. Kim, and K. Kim, "A kinetically controlled molecular switch based on bistable [2]rotaxane," Chem. Commun. 11, 1042-1043 (2001) [doi: 10.1039/b103380h].

[35] G. Kaiser, T. Jarrosson, S. Otto, Y. F. Ng, A. D. Bond, and J. K. M. Sanders, "Lithiumtemplated synthesis of a donor-acceptor pseudorotaxane and catenane," Angew. Chem. Int. Ed. 43, 1959-1962 (2004) [doi:10.1002/anie.200353075].

[36] V. Bermudez, N. Capron, T. Gase, F. G. Gatti, F. Kajzar, D. A. Leigh, F. Zerbetto, and S. W. Zhang, "Influencing intramolecular motion with an alternating electric field," Nature 406, 608-611 (2000) [doi:10.1038/35020531]. 
[37] R. Ballardini, V. Balzani, M. T. Gandolfi, L. Prodi, M. Venturi, D. Philp, H. G. Ricketts, and J. F. Stoddart, "A photochemically driven molecular machine," Angew. Chem. Int. Ed. 32, 1301-1303 (1993) [doi:10.1002/anie.199313011].

[38] S. Saha and J. F. Stoddart, "Photo-driven molecular devices," Chem. Soc. Rev. 36, 7792 (2007) [doi:10.1039/b607187b].

[39] X. Ma, Q. C. Wang, and H. Tian, "Photo-driven molecular shuttles," Prog. Chem. 21, 106-115 (2009).

[40] X. Ma and H. Tian, "Bright functional rotaxanes," Chem. Soc. Rev. 39, 70-80 (2010) [doi:10.1039/b901710k].

[41] V. Balzani, M. Clemente-Leon, A. Credi, M. Semeraro, M. Venturi, H. R. Tseng, S. Wenger, S. Saha, and J. F. Stoddart, "A comparison of shuttling mechanisms in two constitutionally isomeric bistable rotaxane-based sunlight-powered nanomotors," Austral. J. Chem. 59, 193-206 (2006) [doi:10.1071/CH06019].

[42] P. Mobian, J. M. Kern, and J. P. Sauvage, "Light-driven machine prototypes based on dissociative excited states: photoinduced decoordination and thermal recoordination of a ring in a ruthenium(II)-containing [2]catenane," Angew. Chem. Int. Ed. 43, 23922395 (2004) [doi:10.1002/anie.200352522].

[43] T. Muraoka, K. Kinbara, Y. Kobayashi, and T. Aida, "Light-driven open-close motion of chiral molecular scissors," J. Am. Chem. Soc. 125, 5612-5613 (2003) [doi:10.1021/ja034994f].

[44] V. Balzani, M. Gomez-Lopez, and J. F. Stoddart, "Molecular machines," Acc. Chem. Res. 31, 405-414 (1998) [doi:10.1021/ar970340y].

[45] M. C. Petty, Langmuir-Blodgett Films: An Introduction, Cambridge University Press, Cambridge, UK (1996) [doi:10.1017/CBO9780511622519].

[46] C. P. Collier, G. Mattersteig, E. W. Wong, Y. Luo, K. Beverly, J. Sampaio, F. M. Raymo, J. F. Stoddart, and J. R. Heath, "A [2]catenane-based solid state electronically reconfigurable switch," $\quad$ Science $\mathbf{2 8 9}, \quad 1172-1175 \quad$ (2000) [doi:10.1126/science.289.5482.1172].

[47] A. Ulman, Characterization of Organic Thin Films, Butterworth-Heinemann, Boston (1995).

[48] Y. B. Zheng, Y. W. Yang, L. Jensen, L. Fang, B. K. Juluri, A. H. Flood, P. S. Weiss, J. F. Stoddart, and T. J. Huang, "Active molecular plasmonics: controlling plasmon resonances with molecular switches," Nano Lett. 9, 819-825 (2009) [doi: $10.1021 / \mathrm{nl} 803539 \mathrm{~g}$ ].

[49] Y. Liu, A. H. Flood, P. A. Bonvallett, S. A. Vignon, B. H. Northrop, H. R. Tseng, J. O. Jeppesen, T. J. Huang, B. Brough, M. Baller, S. Magonov, S. D. Solares, W. A. Goddard, C. M. Ho, and J. F. Stoddart, "Linear artificial molecular muscles," J. Am. Chem. Soc. 127, 9745-9759 (2005) [doi:10.1021/ja051088p].

[50] B. K. Juluri, A. S. Kumar, Y. Liu, T. Ye, Y. W. Yang, A. H. Flood, L. Fang, J. F. Stoddart, P. S. Weiss, and T. J. Huang, "A mechanical actuator driven electrochemically by artificial molecular muscles," ACS Nano 3, 291-300 (2009) [doi:10.1021/nn8002373].

[51] T. J. Huang, A. H. Flood, B. Brough, Y. Liu, P. A. Bonvallet, S. S. Kang, C. W. Chu, T. F. Guo, W. X. Lu, Y. Yang, J. F. Stoddart, and C. M. Ho, "Understanding and harnessing biomimetic molecular machines for NEMS actuation materials," IEEE Trans. Autom. Sci. Eng. 3, 254-259 (2006) [doi:10.1109/TASE.2006.875543].

[52] T. J. Huang, B. Brough, C. M. Ho, Y. Liu, A. H. Flood, P. A. Bonvallet, H. R. Tseng, J. F. Stoddart, M. Baller, and S. Magonov, "A nanomechanical device based on linear molecular motors," Appl. Phys. Lett. 85, 5391-5393 (2004) [doi:10.1063/1.1826222].

[53] T. J. Huang, "Recent developments in artificial molecular-machine-based active nanomaterials and nanosystems," MRS Bull. 33, 226-231 (2008). 
[54] H. R. Tseng, D. M. Wu, N. X. L. Fang, X. Zhang, and J. F. Stoddart, "The metastability of an electrochemically controlled nanoscale machine on gold surfaces," Chem. Phys. Chem. 5, 111-116 (2004) [doi:10.1002/cphc.200300992].

[55] P. S. Weiss, "Functional molecules and assemblies in controlled environments: formation and measurements," Acc. Chem. Res. 41, 1772-1781 (2008) [doi:10.1021/ar8001443].

[56] M. J. Shuster, A. Vaish, M. E. Szapacs, M. E. Anderson, P. S. Weiss, and A. M. Andrews, "Biospecific recognition of tethered small molecules diluted is selfassembled monolayers," Adv. Mater. 20, 164-167 (2008) [doi:10.1002/adma.200700082].

[57] A. M. Moore, B. A. Mantooth, Z. J. Donhauser, Y. X. Yao, J. M. Tour, and P. S. Weiss, "Real-time measurements of conductance switching and motion of single oligo(phenylene ethynylene) molecules," J. Am. Chem. Soc. 129, 10352-10353 (2007) [doi:10.1021/ja0745153].

[58] A. M. Moore and P. S. Weiss, "Functional and spectroscopic measurements with scanning tunneling microscopy," Annu. Rev. Anal. Chem. 1, 857-882 (2008) [doi:10.1146/annurev.anchem.1.031207.112932].

[59] A. S. Kumar, T. Ye, T. Takami, B. C. Yu, A. K. Flatt, J. M. Tour, and P. S. Weiss, "Reversible photo-switching of single azobenzene molecules in controlled nanoscale environments," Nano Lett. 8, 1644-1648 (2008) [doi:10.1021/nl080323+].

[60] C. A. Nijhuis, B. J. Ravoo, J. Huskens, and D. N. Reinhoudt, "Electrochemically controlled supramolecular systems," Coord. Chem. Rev. 251, 1761-1780 (2007) [doi:10.1016/j.ccr.2007.02.001].

[61] T. B. Lu, L. Zhang, G. W. Gokel, and A. E. Kaifer, "The first surface-attached catenane - self-assembly of a 2-component monolayer," J. Am. Chem. Soc. 115, $2542-$ 2543 (1993) [doi:10.1021/ja00059a078].

[62] R. Klajn, L. Fang, A. Coskun, M. A. Olson, P. J. Wesson, J. F. Stoddart, and B. A. Grzybowski, "Metal nanoparticles functionalized with molecular and supramolecular switches," J. Am. Chem. Soc. 131, 4233-4235 (2009) [doi:10.1021/ja9001585].

[63] A. L. Vance, T. M. Willey, T. van Buuren, A. J. Nelson, C. Bostedt, G. A. Fox, and L. J. Terminello, "XAS and XPS characterization of a surface-attached rotaxane," Nano Lett. 3, 81-84 (2003) [doi:10.1021/n1025814n].

[64] E. H. Witlicki, S. W. Hansen, M. Christensen, T. S. Hansen, S. D. Nygaard, J. O. Jeppesen, E. W. Wong, L. Jensen, and A. H. Flood, "Determination of binding strengths of a host-guest complex using resonance Raman scattering," J. Phys. Chem. A 113, 9450-9457 (2009) [doi:10.1021/jp905202x].

[65] J. B. Jackson and N. J. Halas, "Surface-enhanced Raman scattering on tunable plasmonic nanoparticle substrates," Proc. Natl. Acad. Sci. USA 101, 17930-17935 (2004) [doi:10.1073/pnas.0408319102].

[66] E. H. Witlicki, S. S. Andersen, S. W. Hansen, J. O. Jeppesen, E. W. Wong, L. Jensen, and A. H. Flood, "Turning on resonant SERRS using the chromophore-plasmon coupling created by host-guest complexation at a plasmonic nanoarray," J. Am. Chem. Soc. 132, 6099-6107 (2010) [doi:10.1021/ja910155b].

[67] I. Pockrand, J. D. Swalen, J. G. Gordon, and M. R. Philpott, "Surface-plasmon spectroscopy of organic monolayer assemblies," Surf. Sci. 74, 237-244 (1978) [doi:10.1016/0039-6028(78)90283-2].

[68] S. K. Gray, "Surface plasmon-enhanced spectroscopy and photochemistry," Plasmonics 2, 143-146 (2007) [doi:10.1007/s11468-007-9038-7].

[69] H. Shigekawa, K. Miyake, J. Sumaoka, A. Harada, and M. Komiyama, "The molecular abacus: STM manipulation of cyclodextrin necklace," J. Am. Chem. Soc. 122, 54115412 (2000) [doi:10.1021/ja000037j]. 
[70] J. Berna, D. A. Leigh, M. Lubomska, S. M. Mendoza, E. M. Perez, P. Rudolf, G. Teobaldi, and F. Zerbetto, "Macroscopic transport by synthetic molecular machines," Nat. Mater. 4, 704-710 (2005) [doi:10.1038/nmat1455].

[71] K. Patel, S. Angelos, W. R. Dichtel, A. Coskun, Y. W. Yang, J. I. Zink, and J. F. Stoddart, "Enzyme-responsive snap-top covered silica nanocontainers," J. Am. Chem. Soc. 130, 2382-2383 (2008) [doi:10.1021/ja0772086].

[72] S. Angelos, Y. W. Yang, K. Patel, J. F. Stoddart, and J. I. Zink, "pH-responsive supramolecular nanovalves based on cucurbit[6]uril pseudorotaxanes," Angew. Chem. Int. Ed. 47, 2222-2226 (2008) [doi:10.1002/anie.200705211].

[73] S. Angelos, N. M. Khashab, Y. W. Yang, A. Trabolsi, H. A. Khatib, J. F. Stoddart, and J. I. Zink, "pH clock-operated mechanized nanoparticles," J. Am. Chem. Soc. 131, 12912-12914 (2009) [doi:10.1021/ja9010157].

[74] K. K. Coti, M. E. Belowich, M. Liong, M. W. Ambrogio, Y. A. Lau, H. A. Khatib, J. I. Zink, N. M. Khashab, and J. F. Stoddart, "Mechanised nanoparticles for drug delivery," Nanoscale 1, 16-39 (2009) [doi:10.1039/b9nr00162j].

[75] N. G. Liu, D. R. Dunphy, P. Atanassov, S. D. Bunge, Z. Chen, G. P. Lopez, T. J. Boyle, and C. J. Brinker, "Photoregulation of mass transport through a photoresponsive azobenzene-modified nanoporous membrane," Nano Lett. 4, 551-554 (2004) [doi:10.1021/n10350783].

[76] D. P. Ferris, Y. L. Zhao, N. M. Khashab, H. A. Khatib, J. F. Stoddart, and J. I. Zink, "Light-operated mechanized nanoparticles," J. Am. Chem. Soc. 131, 1686-1688 (2009) [doi:10.1021/ja807798g].

[77] S. A. Kalele, N. R. Tiwari, S. W. Gosavi, and S. K. Kulkarni, "Plasmon-assisted photonics at the nanoscale," J. Nanophoton. 1, 012501 (2007) [doi:10.1117/1.2748429].

[78] M. A. Motyka and A. Lakhtakia, "Multiple trains of same-color surface plasmonpolaritons guided by the planar interface of a metal and a sculptured nematic thin film," J. Nanophoton. 2, 021910 (2008) [doi:10.1117/1.3033757].

[79] A. Lakhtakia, "Toward optical sensing of metal nanoparticles using chiral sculptured thin films," J. Nanophoton. 1, 019502 (2007) [doi:10.1117/1.2753364].

[80] A. Lakhtakia, M. W. McCall, J. A. Sherwin, Q. H. Wu, and I. J. Hodgkinson, "Sculptured-thin-film spectral holes for optical sensing of fluids," Opt. Commun. 194, 33-46 (2001) [doi:10.1016/S0030-4018(01)01225-1].

[81] V. M. Shalaev and S. Kawata, Nanophotonics with Surface Plasmons, Elsevier, Oxford, UK (2007).

[82] S. Lal, S. Link, and N. J. Halas, "Nano-optics from sensing to waveguiding," Nat. Photon. 1, 641-648 (2007) [doi:10.1038/nphoton.2007.223].

[83] S. Saha, E. Johansson, A. H. Flood, H. R. Tseng, J. I. Zink, and J. F. Stoddart, "A photoactive molecular triad as a nanoscale power supply for a supramolecular machine," Chem. Eur. J. 11, 6846-6858 (2005) [doi:10.1002/chem.200500371].

[84] M. Granmar and A. Cho, "Technology-electronic paper: a revolution about to unfold?" Science 308, 785-786 (2005) [doi:10.1126/science.308.5723.785].

[85] B. Comiskey, J. D. Albert, H. Yoshizawa, and J. Jacobson, "An electrophoretic ink for all-printed reflective electronic displays," Nature 394, 253-255 (1998) [doi:10.1038/28349].

[86] R. A. Hayes and B. J. Feenstra, "Video-speed electronic paper based on electrowetting," Nature 425, 383-385 (2003) [doi:10.1038/nature01988].

[87] T. Ikeda and J. F. Stoddart, "Electrochromic materials using mechanically interlocked molecules," Sci. Technol. Adv. Mater. 9, 014104 (2008) [doi:10.1088/14686996/9/1/014104].

[88] D. W. Steuerman, H. R. Tseng, A. J. Peters, A. H. Flood, J. O. Jeppesen, K. A. Nielsen, J. F. Stoddart, and J. R. Heath, "Molecular-mechanical switch-based solid- 
state electrochromic devices," Angew. Chem. Int. Ed. 43, 6486-6491 (2004) [doi:10.1002/anie.200461723].

[89] T. Ikeda, I. Aprahamian, and J. F. Stoddart, "Blue-colored donor-acceptor [2]rotaxane," Org. Lett. 9, 1481-1484 (2007) [doi:10.1021/o1070170h].

[90] T. Ikeda, S. Saha, I. Aprahamian, K. C. F. Leung, A. Williams, W. Q. Deng, A. H. Flood, W. A. Goddard, and J. F. Stoddart, "Toward electrochemically controllable tristable three-station [2]catenanes," Chem. Asian J. 2, 76-93 (2007) [doi:10.1002/asia.200600355].

[91] Y. Chen, J. Au, P. Kazlas, A. Ritenour, H. Gates, and M. McCreary, "Flexible activematrix electronic ink display," Nature 423, 136-136 (2003) [doi:10.1038/423136a].

[92] J. A. Rogers, "Electronics-toward paperlike displays," Science 291, 1502-1503 (2001) [doi:10.1126/science.291.5508.1502].

[93] M. L. Brongersma and P. G. Kik, Surface Plasmon Nanophotonics, Springer, Berlin (2007) [doi:10.1007/978-1-4020-4333-8].

[94] H. A. Atwater, S. Maier, A. Polman, J. A. Dionne, and L. Sweatlock, "The new "p-n junction: plasmonics enables photonic access to the nanoworld," MRS Bull. 30, 385389 (2005).

[95] A. V. Zayats, Smolyaninov, II, and A. A. Maradudin, "Nano-optics of surface plasmon polaritons," Phys. Rep. 408, 131-314 (2005) [doi:10.1016/j.physrep.2004.11.001].

[96] P. Berini, R. Charbonneau, and N. Lahoud, "Long-range surface plasmons on ultrathin membranes," Nano Lett. 7, 1376-1380 (2007) [doi:10.1021/n1070464w].

[97] S. I. Bozhevolnyi, V. S. Volkov, E. Devaux, J. Y. Laluet, and T. W. Ebbesen, "Channel plasmon subwavelength waveguide components including interferometers and ring resonators," Nature 440, 508-511 (2006) [doi:10.1038/nature04594].

[98] S. A. Maier, S. R. Andrews, L. Martin-Moreno, and F. J. Garcia-Vidal, "Terahertz surface plasmon-polariton propagation and focusing on periodically corrugated metal wires," Phys. Rev. Lett. 97, 176805 (2006) [doi:10.1103/PhysRevLett.97.176805].

[99] B. Steinberger, A. Hohenau, H. Ditlbacher, A. L. Stepanov, A. Drezet, F. R. Aussenegg, A. Leitner, and J. R. Krenn, "Dielectric stripes on gold as surface plasmon waveguides," Appl. Phys. Lett. 88, 094104 (2006) [doi:10.1063/1.2180448].

[100] J. C. Weeber, M. U. Gonzalez, A. L. Baudrion, and A. Dereux, "Surface plasmon routing along right angle bent metal strips," Appl. Phys. Lett. 87, 221101 (2005) [doi:10.1063/1.2130393].

[101] F. Lopez-Tejeira, S. G. Rodrigo, L. Martin-Moreno, F. J. Garcia-Vidal, E. Devaux, T. W. Ebbesen, J. R. Krenn, I. P. Radko, S. I. Bozhevolnyi, M. U. Gonzalez, J. C. Weeber, and A. Dereux, "Efficient unidirectional nanoslit couplers for surface plasmons," Nat. Phys. 3, 324-328 (2007) [doi:10.1038/nphys584].

[102] M. W. Knight, N. K. Grady, R. Bardhan, F. Hao, P. Nordlander, and N. J. Halas, "Nanoparticle-mediated coupling of light into a nanowire," Nano Lett.7, 2346-2350 (2007) [doi:10.1021/n1071001t].

[103] J. H. Dai, F. Cajko, I. Tsukerman, and M. I. Stockman, "Electrodynamic effects in plasmonic nanolenses," Phys. Rev. B 77, $115419 \quad$ (2008) [doi:10.1103/PhysRevB.77.115419].

[104] D. R. Smith, J. B. Pendry, and M. C. K. Wiltshire, "Metamaterials and negative refractive index," Science 305, 788-792 (2004) [doi:10.1126/science.1096796].

[105] Y. Leroux, J. C. Lacroix, C. Fave, V. Stockhausen, N. Felidj, J. Grand, A. Hohenau, and J. R. Krenn, "Active plasmonic devices with anisotropic optical response: a step toward active polarizer," Nano Lett. 9, 2144-2148 (2009) [doi:10.1021/n1900695j].

[106] A. V. Krasavin and N. I. Zheludev, "Active plasmonics: controlling signals in $\mathrm{Au} / \mathrm{Ga}$ waveguide using nanoscale structural transformations," Appl. Phys. Lett. 84, 14161418 (2004) [doi:10.1063/1.1650904]. 
[107] J. Dintinger, S. Klein, and T. W. Ebbesen, "Molecule-surface plasmon interactions in hole arrays: Enhanced absorption, refractive index changes, and all-optical switching," Adv. Mater. 18, 1267-1270 (2006) [doi:10.1002/adma.200502393].

[108] J. Lee, P. Hernandez, J. Lee, A. O. Govorov, and N. A. Kotov, "Exciton-plasmon interactions inmolecular spring assemblies of nanowires and wavelength-based protein detection," Nat. Mater. 6, 291-295 (2007) [doi:10.1038/nmat1869].

[109] G. L. Liu, Y. T. Long, Y. Choi, T. Kang, and L. P. Lee, "Quantized plasmon quenching dips nanospectroscopy via plasmon resonance energy transfer," Nat. Methods 4, 1015-1017 (2007) [doi:10.1038/nmeth1133].

[110] A. J. Haes, S. L. Zou, J. Zhao, G. C. Schatz, and R. P. Van Duyne, "Localized surface plasmon resonance spectroscopy near molecular resonances," J. Am. Chem. Soc. 128, 10905-10914 (2006) [doi:10.1021/ja063575q].

[111] G. A. Wurtz, P. R. Evans, W. Hendren, R. Atkinson, W. Dickson, R. J. Pollard, A. V. Zayats, W. Harrison, and C. Bower, "Molecular plasmonics with tunable excitonplasmon coupling strength in J-aggregate hybridized Au nanorod assemblies," Nano Lett. 7, 1297-1303 (2007) [doi:10.1021/n1070284m].

[112] G. P. Wiederrecht, G. A. Wurtz, and J. Hranisavljevic, "Coherent coupling of molecular excitons to electronic polarizations of noble metal nanoparticles," Nano Lett. 4, 2121-2125 (2004) [doi:10.1021/n10488228].

[113] Y. Sugawara, T. A. Kelf, J. J. Baumberg, M. E. Abdelsalam, and P. N. Bartlett, "Strong coupling between localized plasmons and organic excitons in metal nanovoids," Phys. Rev. Lett. 97, 266808 (2006) [doi:10.1103/PhysRevLett.97.266808].

[114] N. T. Fofang, T. H. Park, O. Neumann, N. A. Mirin, P. Nordlander, and N. J. Halas, "Plexcitonic nanoparticles: plasmon-exciton coupling in nanoshell-J-aggregate complexes," Nano Lett. 8, 3481-3487 (2008) [doi:10.1021/nl8024278].

[115] T. Ambjornsson, G. Mukhopadhyay, S. P. Apell, and M. Kall, "Resonant coupling between localized plasmons and anisotropic molecular coatings in ellipsoidal metal nanoparticles," Phys. Rev. B 73, 085412 (2006) [doi:10.1103/PhysRevB.73.085412].

[116] Y. B. Zheng, B. K. Juluri, X. L. Mao, T. R. Walker, and T. J. Huang, "Systematic investigation of localized surface plasmon resonance of long-range ordered $\mathrm{Au}$ nanodisk arrays," J. Appl. Phys. 103, 014308 (2008) [doi:10.1063/1.2828146].

[117] V. K. S. Hsiao, Y. B. Zheng, B. K. Juluri, and T. J. Huang, "Light-driven plasmonic switches based on Au nanodisk arrays and photoresponsive liquid crystals," Adv. Mater. 20, 3528-3532 (2008) [doi:10.1002/adma.200800045].

[118] Y. Leroux, J. C. Lacroix, C. Fave, G. Trippe, N. Felidj, J. Aubard, A. Hohenau, and J. R. Krenn, "Tunable electrochemical switch of the optical properties of metallic nanoparticles," ACS Nano 2, 728-732 (2008) [doi:10.1021/nn700438a].

[119] P. A. Kossyrev, A. J. Yin, S. G. Cloutier, D. A. Cardimona, D. H. Huang, P. M. Alsing, and J. M. Xu, "Electric field tuning of plasmonic response of nanodot array in liquid crystal matrix," Nano Lett. 5, 1978-1981 (2005) [doi:10.1021/n10513535].

[120] R. Chapman and P. Mulvaney, "Electro-optical shifts in silver nanoparticle films," Chem. Phys. Lett. 349, 358-362 (2001) [doi:10.1016/S0009-2614(01)01145-9].

[121] W. P. Hall, J. N. Anker, Y. Lin, J. Modica, M. Mrksich, and R. P. Van Duyne, "A calcium-modulated plasmonic switch," J. Am. Chem. Soc. 130, 5836-5837 (2008) [doi:10.1021/ja7109037].

[122] C. Sonnichsen, B. M. Reinhard, J. Liphardt, and A. P. Alivisatos, "A molecular ruler based on plasmon coupling of single gold and silver nanoparticles," Nat. Biotechnol. 23, 741-745 (2005) [doi:10.1038/nbt1100].

[123] G. L. Liu, Y. D. Yin, S. Kunchakarra, B. Mukherjee, D. Gerion, S. D. Jett, D. G. Bear, J. W. Gray, A. P. Alivisatos, L. P. Lee, and F. Q. F. Chen, "A nanoplasmonic molecular ruler for measuring nuclease activity and DNA footprinting," Nat. Nanotechnol. 1, 47-52 (2006) [doi:10.1038/nnano.2006.51]. 
[124] B. M. Reinhard, S. Sheikholeslami, A. Mastroianni, A. P. Alivisatos, and J. Liphardt, "Use of plasmon coupling to reveal the dynamics of DNA bending and cleavage by single EcoRV restriction enzymes," Proc. Natl. Acad. Sci. USA 104, 2667-2672 (2007) [doi:10.1073/pnas.0607826104].

[125] T. Ye, A. S. Kumar, S. Saha, T. Takami, T. J. Huang, J. F. Stoddart, and P. S. Weiss, "Changing stations in single bistable rotaxane molecules under electrochemical control," ACS Nano 4, 3697-3701 (2010) [doi:10.1021/nn100545r].

[126] D. Li, R. H. Baughman, T. J. Huang, J. F. Stoddart, and P. S. Weiss, "Molecular, supramolecular, and macromolecular motors and artificial muscles," MRS Bull. 34, 671-681 (2009).

[127] V. K. S. Hsiao, John R. Waldeisen, and T. J. Huang, "Aminopropyltriethoxysilane (APTES)-functionalized nanoporous polymeric gratings: fabrication and application in biosensing," J. Mater. Chem. 17, 4896-4901 (2007) [doi: 10.1039/b711200a].

[128] T. J. Huang and B. K. Juluri, "Biological and biomimetic molecular machines," Nanomedicine 3, 107-124 (2008) [doi:10.2217/17435889.3.1.107].

[129] W. Yan, V. K. S. Hsiao, Y. B. Zheng, Y. M. Shariff, T. Gao, and T. J. Huang, "Towards nanoporous polymer thin film-based drug delivery systems," Thin Solid Films 517, 1794-1798 (2009) [doi:10.1016/j.tsf.2008.09.080].

[130] Y. B. Zheng, T. J. Huang, A. Y. Desai, S. J. Wang, L. K. Tan, H. Gao, and A. C. H. Huan, "Thermal behavior of localized surface plasmon resonance of $\mathrm{Au} / \mathrm{TiO}_{2}$ core/shell nanoparticle arrays," Appl. Phys. Lett. 90, 183117 (2007) [doi:10.1063/1.2736283]

[131] Y. J. Liu, Y. B. Zheng, J. Shi, H. Huang, T. R. Walker, and T. J. Huang, "Optically switchable gratings based on azo-dye-doped, polymer-dispersed liquid crystals," Opt. Lett. 34, 2351-2353 (2009) [doi:10.1364/OL.34.002351].

[132] B. K. Juluri, M. Lu, Y. B. Zheng, L. Jensen, and T. J. Huang, "Coupling between molecular and plasmonic resonances: effect of molecular absorbance," J. Phys. Chem. C 113, 18499-18503 (2009) [doi:10.1021/jp908215a].

[133] Y. B. Zheng, B. K. Juluri, L. L. Jensen, D. Ahmed, M. Lu, L. Jensen, and T. J. Huang, "Dynamic tuning of plasmon-exciton coupling in arrays of nanodisk-J-aggregate complexes," Adv. Mater. 22, 3603-3607 (2010) [doi:10.1002/adma.201000251].

Yue Bing Zheng received his Ph.D. in Engineering Science and Mechanics from The Pennsylvania State University in 2010 under the supervision of Prof. Tony Jun Huang, his M.S. in Physics from National University of Singapore in 2004, and his B.S. in Physics from Nankai University in 2001. His research interests include molecular machinery, molecular electronics, nanophotonics, plasmonics, nanomedicine, functional supramolecular assemblies, nanofabrication, and scanning tunneling microscopy. He has authored or coauthored over 30 journal publications and 2 book chapters in these fields. Currently, he is a postdoctoral fellow in Prof. Paul S. Weiss's group at the University of California, Los Angeles (UCLA).

Qingzhen Hao is currently a Ph.D candidate in Prof. Tony Jun Huang's group at The Pennsylvania State University. He received his B.S. in physics from Hong Kong Baptist University in 2006. His research interest is on plasmonic metallic nanostructures. The nanofabrication, characterization, active control, and applications of those nanostructures are his main research focus.

Ying-Wei Yang is a postdoctoral fellow at the University of California, Irvine. He received his B. Sc. in chemistry from Nankai University in 2000 and his Ph.D. degree in physical chemistry from the same university in 2005 under the supervision of Professor Yu Liu. He 
joined Professor Fraser Stoddart's research group at University of California, Los Angeles (UCLA) as a postdoctoral scholar in August 2006. At UCLA, he conducted research in the field of organic chemistry and material science, with emphasis on biocompatible mechanized nanoparticles for biomedical application and mechanically interlocked molecule-based nanomaterials for NEMS and Nanophotonics. He is the author of more than 30 journal papers. His current research interests include molecular recognition and self-assembly, new catalyst design for efficient synthesis of macromolecules, and the development of new materials for microfluidic device fabrication.

Brian Kiral is a graduate student in his first year at The Pennsylvania State University pursuing a Ph.D. in engineering science. He received his B.S. from the Rose-Hulman Institute of Technology in engineering physics. His research interests are in plasmonics/nanophotonics and include conventional metallic plasmonic nanostructures and plasmonically enhanced solid state devices. He is interested in the fabrication, characterization, and theoretical simulation of these structures.

I-Kao Chiang received his B.S. from The Pennsylvania State University majoring in engineering science and minoring nanotechnology. He will be a first-year graduate student at the University of Pennsylvania pursuing a master degree in computer science. His areas of interest are microfluidics and computer vision \& graphics. These areas include on-chip detection, manipulation, simulation, and imaging.

Tony Jun Huang is an Associate Professor in the Department of Engineering Science and Mechanics at The Pennsylvania State University. He received his Ph.D. degree in Mechanical and Aerospace Engineering from the University of California, Los Angeles (UCLA) in 2005, and his B.S. and M.S. degrees in Energy and Power Engineering from Xi'an Jiaotong University, Xi'an, China, in 1996 and 1999, respectively. His research is focused on (1) multi-physics of active nanostructures, and (2) multi-physics of micro/nano fluidics and labon-a-chip systems, including acoustic microfluidics and optofluidics. He has authored or coauthored over 100 technical publications and several book chapters in these fields. $\mathrm{He}$ serves as Vice Chair of the American Society of Mechanical Engineers (ASME) Nanotechnology Council, chair of the ASME Society-Wide Micro/Nano Technology Forum, and member of editorial board for Journal of Nanomedicine \& Biotherapeutic Discovery and Journal of Nanoscience Letters. More information about him and his research group can be found at www.esm.psu.edu/huang/. 